\title{
An Algorithm for LES of Premixed Compressible Flows Using the Conditional Moment Closure Model
}

\author{
B. Thornber ${ }^{\mathrm{a}, \mathrm{b}}$, R.W. Bilger ${ }^{\mathrm{b}}$, A. R. Masri ${ }^{\mathrm{b}}$, E. R. Hawkes ${ }^{\mathrm{c}}$ \\ ${ }^{a}$ Fluid Mechanics and Computational Science Group, Dept. Aerospace Science, Cranfield \\ University, UK \\ ${ }^{b}$ Thermofluids, School of Aerospace, Mechanical and Mechatronic Engineering, The \\ University of Sydney,Australia \\ ${ }^{c}$ School of Photovoltaic and Renewable Energy Engineering/School of Mechanical and \\ Manufacturing Engineering, The University of New South Wales, Australia
}

\begin{abstract}
A novel numerical method has been developed to couple a recent high order accurate fully compressible upwind method with the Conditional Moment Closure combustion model. The governing equations, turbulence modelling and numerical methods are presented in full. The new numerical method is validated against Direct Numerical Simulation (DNS) data for a lean premixed methane slot burner. Although the modelling approaches are based on non-premixed flames and hence not expected to be valid for a wide range of premixed flames, the predicted flame is just $10 \%$ longer than that in the DNS and excellent agreement of mean mass fractions, conditional mass fractions and temperature is demonstrated. This new numerical method provides a very useful framework for future application of CMC to premixed as well as non-premixed combustion.
\end{abstract}

Keywords: Conditional Moment Closure, Large Eddy Simulation, compressible, combustion, premixed, turbulent combustion, non-premixed

\section{Introduction}

With current theoretical knowledge of combustion it is becoming possible to quantitatively predict the structure of turbulent flames through computational simulation $[1,2]$. Recently, Large Eddy Simulation (LES) has emerged as a very promising methodology for simulating complex, turbulent flows. It is not as computationally expensive as Direct Numerical Simulation (DNS) 
yet performs better than Reynolds-Averaged Navier Stokes (RANS) methods in unsteady flows [3]. LES has the advantage in that a significant proportion of the turbulent fluctuations are resolved during the computation, hence reducing the importance of modelling errors $[4,5]$. Despite this, many have observed that LES of combustion is complicated by the strong dependence of reaction rates on the subgrid variation of species concentrations and temperature distributions $[6,7]$.

Combustion modelling aims to address this fundamental problem by providing closure for the filtered mean reaction rates and subgrid scalar fluxes required to solve the governing equations for the filtered chemical species mass fractions and in compressible applications the filtered energy equation. The wide range of potential subgrid reaction rate models for LES are reviewed in detail in Pitsch [3], Poinsot \& Veynante [6], and a summary of their application to premixed flows is given in Lipatnikov \& Chomiak [8]. The Conditional Moment Closure (CMC) model [9-11] has been thoroughly validated in RANS simulations of turbulent non-premixed flames and has recently been successfully extended to LES (denoted LES-CMC) of turbulent diffusion flames and bluff body flames [12-17]. This modelling approach has several advantages, most notably that it provides a simple means to integrate finite rate complex chemistry effects into an LES. Closure is achieved through assuming that the variations around the conditional means are small.

LES-CMC has not yet been applied to premixed combustion, however it has been successfully applied in RANS simulations of a premixed generic gas turbine combustor [18]. A key challenge in premixed applications in CMC is the specification of an appropriate conditioning variable. In non-premixed applications the conditioning variable is usually the mixture fraction, which is well correlated to the overall reaction progress such that conditional fluctuations are small. Bilger [19] suggested a novel marker field which has been employed with success using a laminar flamelet type closure in premixed LES by Duwig and Fuchs [20]. Other typical progress variable choices include scaled sensible enthalpy [18, 21], scaled temperature [6, 22], or scaled mass fractions [23-25].

Using a direct numerical simulation database modelling a turbulent lean premixed methane slot flame due to Sankaran et al. [26], it has been shown in 'a-priori' tests [27] that the first order CMC closure assumption based on mass fraction of $\mathrm{O}_{2}$ gives good results for the reaction rate of the major species. Vreman et al. [28] undertook LES at low Reynolds numbers using an $\mathrm{O}_{2}$ based progress variable gaining good results with very coarse grid resolutions 
for a lean methane Bunsen flame. Therefore, this choice of progress variable is used in the work presented here.

The vast majority of numerical methods chosen for combustion simulations are based on low Mach or incompressible formulations, where pressure fluctuations arise as a solution to the Poisson equation. In incompressible formulations the combustion model usually provides the density directly, as opposed to reality where the density is given by the continuity equation. There are only a handful of LES approaches employing fully compressible methods with more complex models than a simple quasi-laminar assumption (with finite rate chemistry) [22, 24, 29-34]. Of these, only the methods proposed by Berglund et al. [33], Amsden [29], and Molkov et al. [34] are capable of capturing shocks. For unsteady, time-accurate LES there are many cases where compressibility is an important factor in the flow, e.g. deflagrations, detonations, knock or ringing in homogeneous charge compression ignition engines at high load, knocking spark-ignition engines, combustion noise and acoustically driven instabilities in gas turbines.

There is a second strong driver to employ fully compressible codes, namely that they do not require the expensive implicit iterative loops which are present in incompressible algorithms. A typical compressible method will employ explicit time discretisation methods such as Runge-Kutta. This leads naturally to algorithms that are more easily parallelised, scale more efficiently and are straightforward to apply to complex realistic geometries.

This paper presents a new high-order accurate, fully compressible (shock capturing) implementation of the $\mathrm{CMC}$ modelling approach for lean premixed turbulent combustion. The CMC model is coupled through the energy and species equations using a fifth-order accurate in space modified Godunov method which has recently been derived specifically for problems including both compressible features and turbulent mixing [35, 36]. This paper also presents the first application of LES-CMC to unsteady premixed combustion. In addition, the same proposed algorithmic structure can be used for nonpremixed combustion using LES-CMC. The proposed algorithm is tested against the slot flame DNS of Sankaran et al. [26].

\section{Governing Equations}

\subsection{LES Equations for a Compressible Reacting Flow}

This subsection presents the governing equations for an LES of a compressible, reacting mixture $[6,37]$. These consist of the continuity equation 


$$
\frac{\partial \bar{\rho}}{\partial t}+\frac{\partial \bar{\rho} \tilde{u}_{i}}{\partial x_{i}}=0
$$

the species conservation equation,

$$
\frac{\partial \bar{\rho} \tilde{Y}_{k}}{\partial t}+\frac{\partial \bar{\rho} \tilde{u}_{i} \tilde{Y}_{k}}{\partial x_{i}}-\frac{\partial \bar{J}_{i k}}{\partial x_{i}}=\underbrace{-\frac{\partial}{\partial x_{i}} \bar{\rho}\left(\widetilde{u_{i} Y_{k}}-\tilde{u}_{i} \tilde{Y}_{k}\right)}_{S_{1}}+\underbrace{\bar{\rho} \tilde{\dot{w}}_{k}}_{S_{2}}
$$

where,

$$
\bar{J}_{i k}=\overline{\rho\left(D_{k} \frac{\partial Y_{k}}{\partial x_{i}}-Y_{k} \sum_{j=1}^{N} D_{j} \frac{\partial Y_{j}}{\partial x_{i}}\right)} \approx \bar{\rho}\left(\bar{D}_{k} \frac{\partial \tilde{Y}_{k}}{\partial x_{i}}-\tilde{Y}_{k} \sum_{j=1}^{N} \bar{D}_{j} \frac{\partial \tilde{Y}_{j}}{\partial x_{i}}\right)
$$

where $\rho$ is the density, $u_{i}$ are the velocity components, $Y_{k}$ are the species mass fractions, $J_{k}$ is the species diffusive transport term (assumed Fickian here), $\dot{w}_{K}$ is the mass fraction production rate due to chemical reaction, (.) indicates a Favre filtered value and $\overline{(.)}$ indicates a spatially filtered value. Next, the filtered momentum equation becomes,

$$
\frac{\partial \bar{\rho} \tilde{u}_{i}}{\partial t}+\frac{\partial \bar{\rho} \tilde{u}_{i} \tilde{u}_{j}}{\partial x_{i}}+\frac{\partial \bar{p}}{\partial x_{i}}-\frac{\partial \hat{\sigma}_{i j}}{\partial x_{j}}=\underbrace{-\frac{\partial \tau_{i j}}{\partial x_{i}}}_{A_{1}}+\underbrace{\frac{\partial}{\partial x_{j}}\left(\bar{\sigma}_{i j}-\hat{\sigma}_{i j}\right)}_{A_{2}},
$$

where $\tau_{i j}=\bar{\rho}\left(\widetilde{u_{i} u_{j}}-\tilde{u}_{i} \tilde{u}_{j}\right)$ is the Reynolds stress tensor, $\sigma_{i j}$ is the viscous stress tensor, $\hat{\sigma}_{i j}=\sigma_{i j}\left(\partial \tilde{u}_{i} / \partial x_{j}, \tilde{T}\right)$ and $p$ is the pressure.

Finally, the energy equation employed governs the evolution of sensible plus kinetic energy, sometimes denoted as 'total non-chemical energy',

$$
\begin{aligned}
& \frac{\partial \widehat{\rho E}}{\partial t}+\frac{\partial \tilde{u}_{i}(\widehat{\rho E}+\bar{p})}{\partial x_{i}}+\frac{\partial \hat{q}_{j}}{\partial x_{j}}-\frac{\partial}{\partial x_{j}}\left(\hat{\sigma}_{i j} \tilde{u}_{i}\right)= \\
& \underbrace{\frac{\partial}{\partial x_{i}} \overline{\left(\rho \sum_{k=1}^{N} h_{s, k} Y_{k} J_{k}\right)}}_{R_{1}}+\underbrace{\overline{\dot{w}_{T}}}_{R_{2}}-B_{1}-B_{2}-B_{3}+B_{4}+B_{5}+B_{6}-B_{7},
\end{aligned}
$$

where $\widehat{\rho E}=\bar{\rho} \tilde{e}+1 / 2 \bar{\rho} \tilde{u}_{i} \tilde{u}_{i}, \overline{\dot{w}_{T}}=\overline{-\sum_{k=1}^{N} \Delta h_{f, k}^{\circ} \rho \dot{w}_{k}}$ is the heat release due to chemical reaction, $q_{i}=-\lambda\left(\partial T / \partial x_{i}\right)$ where $\lambda$ is the thermal conductivity, 
and $\hat{q}_{i}=q_{i}\left(\partial \tilde{T} / \partial x_{i}\right)$. The terms on the left hand side are computable from the LES flow field, whereas the ones on the right hand side are not. These are defined as:

$$
\begin{aligned}
& B_{1}=\frac{\partial}{\partial x_{i}}\left(\bar{\rho} \widetilde{e_{s} u_{i}}-\overline{\rho e_{s}} \tilde{u}_{i}\right), \quad B_{2}=\overline{p \frac{\partial u_{i}}{\partial x_{i}}}-\bar{p} \frac{\partial \tilde{u}_{i}}{\partial x_{i}}, \quad B_{3}=\frac{\partial}{\partial x_{i}}\left(\tau_{i j} \tilde{u}_{i}\right) \\
& B_{4}=\tau_{i j} \frac{\partial \tilde{u}_{i}}{\partial x_{i}}, \quad B_{5}+B_{6}=\frac{\partial}{\partial x_{j}}\left(\overline{\sigma_{i j} u_{i}}-\hat{\sigma}_{i j} \widetilde{u}_{i}\right), \quad B_{7}=\frac{\partial}{\partial x_{i}}\left(\bar{q}_{i}-\hat{q}_{i}\right)
\end{aligned}
$$

Following previous literature it is common to neglect terms $A_{2}, B_{5}, B_{6}$ and $B_{7}[37]$.

In this paper the novel reconstruction method proposed by Thornber et al. $[35,36]$ is employed to model terms $A_{1}, B_{3}$ and $B_{4}$. This numerical method is not kinetic energy conserving. Rather, the reconstruction method is designed to give a leading order dissipation of turbulent kinetic energy proportional to the velocity increment at the cell interface cubed $\left(\Delta u^{3}\right)$ as expected from Kolmogorov's analysis [38]. The improved interpolation approach helps overcome the typical poor high wavenumber performance of standard compressible Godunov methods [39, 40]. It acts as an implicit subgrid model [41-43] whilst naturally stabilising the numerical solution and retaining monotonicity.

As with all LES models this relies on sufficient separation of the large scales from the scales where numerical dissipation acts strongly. In several previous test cases, this numerical method has demonstrated a good ability to represent the dissipation of turbulent kinetic energy [36, 39, 44, 45], most notably in flows requiring excellent resolution of turbulent scalar mixing parameters [46]. Making the assumption that the implicit dissipation of the numerical method is sufficient to model the dissipation of turbulent kinetic energy, then terms referring to the Reynolds stresses are also neglected, i.e. $A_{1}, B_{3}$ and $B_{4}$. This leaves the terms specifically associated with the species turbulent diffusion, reaction rates, and turbulent pressure/internal energy fluxes - $S_{1}, S_{2}, R_{1}, R_{2}, B_{1}$ and $B_{2}$.

In an analysis of premixed and non-premixed methane air flames, Smooke and Giovangigli [47] demonstrated that the terms including enthalpy diffusion could be neglected by comparison to the other terms in the energy equation. Based on this, the term $R_{1}$ is neglected here. 
Term $S_{1}$ is modelled by a turbulent Schmidt number as:

$$
S_{1}=\frac{\partial}{\partial x_{i}} \frac{\bar{\rho} \bar{\nu}_{t}}{\bar{S} c_{k}} \frac{\partial \tilde{Y}_{k}}{\partial x_{i}}
$$

Terms $B_{1}+B_{2}$ are represented by thermal diffusion via the Prandtl analogy

$$
B_{1}+B_{2}=-\frac{\partial}{\partial x_{i}} \frac{\bar{\rho} \bar{\nu}_{t} C_{p}}{P r_{s g s}} \frac{\partial \tilde{T}}{\partial x_{i}}
$$

where $\bar{\nu}_{t}$ is estimated using the Smagorinsky model [48]:

$$
\bar{\nu}_{t}=\left(C_{s} \Delta\right)^{2}\left(2 \bar{S}_{i j} \bar{S}_{i j}\right)^{1 / 2}
$$

and,

$$
S_{i j}=\frac{1}{2}\left(\frac{\partial \tilde{u}_{i}}{\partial x_{j}}+\frac{\partial \tilde{u}_{j}}{\partial x_{i}}\right),
$$

This leaves the terms $S_{2}$ and $R_{2}$, however $R_{2}$ is essentially determined once a model is chosen for $S_{2}$ as $\overline{\dot{w}_{T}}=\overline{-\sum_{k=1}^{N} \Delta h_{f, k}^{\circ} \rho \dot{w}_{k}}=-\sum_{k=1}^{N} \Delta h_{f, k}^{\circ} \bar{\rho} \tilde{\dot{w}}_{k}$. Closure for these terms is achieved through the application of the first order Conditional Moment Closure assumption which is described in full in Section 2.3 .

\subsection{Thermodynamic and Transport Properties}

The thermodynamic properties of the fluid mixture are computed using standard polynomial data fits to the specific heats at constant pressure [49], $\mathrm{C}_{p}$ and knowledge of the individual species molecular weights and formation enthalpies.

The transport properties are based on the analysis of lean premixed flows presented by Smooke and Giovangigli [47] where an empirical relation for the thermal conductivity $\lambda$ and viscosity $\mu$ is given,

$$
\lambda=C_{p} A\left(\frac{T}{T_{0}}\right)^{r}, \quad \mu=\frac{\operatorname{Pr} \lambda}{C_{p}}
$$

where the Prandtl number $\operatorname{Pr}=0.708$, the reference temperature $T_{0}=298 \mathrm{~K}$, and the coefficients $A=2.58^{-5} \mathrm{~kg} /(\mathrm{m}-\mathrm{s})$ and $r=0.7$. 


\subsection{Conditional Moment Closure}

\subsubsection{Governing Equations}

To consistently solve for the chemical reaction zone the Conditional Moment Closure (CMC) equations are employed [9, 10]. This involves solving for each species in physical space, progress variable space and time. Instead of solving a set of mass fraction equations for each species, this is replaced by a set of CMC equations. An outline derivation of the LES-CMC equations is detailed in Appendix A for premixed combustion following the non-premixed derivation detailed by [13], resulting in the following equation for the evolution of conditional mass fractions:

$$
\frac{\partial Q}{\partial t}+\widetilde{\mathbf{u} \mid \zeta} \cdot \nabla Q=\widetilde{\dot{w}_{k} \mid \zeta}-\widetilde{S_{c} \mid \zeta} \frac{\partial Q}{\partial \zeta}+\widetilde{N \mid \zeta} \frac{\partial^{2} Q}{\partial \zeta}+e_{Q}
$$

where $Q=\widetilde{Y_{k} \mid \zeta}$, the scalar dissipation $\tilde{N}=\bar{D}_{c}(\nabla \tilde{c})^{2}$ and. $\mid \zeta$ refers to a mean conditional on $c=\zeta$ where $c$ is the progress variable. $\tilde{P}(\zeta)$ is the Favre weighted Filtered Probability Density Function (FDF), $\widehat{\dot{w}_{k} \mid \zeta}$ is the conditional filtered mass fraction production rate of species $k$, and the conditionally filtered density $\overline{\rho \mid \zeta} . \tilde{S}_{c}$ is the Favre filtered source term present in the equation for the progress variable, which in this paper is the scaled $\mathrm{O}_{2}$ mass fraction production rate. Finally, $e_{Q}$ is given by

$$
\overline{\rho \mid \zeta} \tilde{P}(\zeta) e_{Q}=\nabla \cdot\left[\overline{\rho \mid \zeta}\left(\widetilde{\mathbf{u} \mid \zeta} Q-\widetilde{\left(\mathbf{u} Y_{k}\right) \mid \zeta}\right) \tilde{P}(\zeta)\right] .
$$

This term is modelled using a gradient type approach giving:

$$
\overline{\rho \mid \zeta} \tilde{P}(\zeta) e_{Q}=\frac{\partial}{\partial x_{i}}\left(\tilde{P}(\zeta) \overline{\rho \mid \zeta} \bar{D}_{t} \frac{\partial Q}{\partial x_{i}}\right),
$$

where $\bar{D}_{t}$ is a turbulent diffusivity. Eqn. (13) is solved for each species at each condition along with the Navier-Stokes equations for the mixture plus a single equation for the transport of $\bar{\rho} \tilde{Y}_{O_{2}}$. As the procedure is computationally expensive, usually the LES-CMC equations are solved on a coarser grid than the Navier-Stokes equations, hence

$$
\begin{aligned}
\frac{\partial Q^{*}}{\partial t}+\widetilde{u_{i}^{*} \mid \zeta} \frac{\partial Q^{*}}{\partial x_{i}}=\widetilde{\dot{w}_{k}^{*} \mid \zeta}+\widetilde{N^{*} \mid \zeta} \frac{\partial^{2} Q^{*}}{\partial \zeta^{2}}+ \\
\\
\quad \frac{1}{\tilde{P}^{*}(\zeta) \overline{\rho^{*} \mid \zeta}} \frac{\partial}{\partial x_{i}}\left(\tilde{P}^{*}(\zeta) \overline{\rho^{*} \mid \zeta} \bar{D}_{t}^{*} \frac{\partial Q^{*}}{\partial x_{i}}\right)-\widetilde{S_{c}^{*} \mid \zeta} \frac{\partial Q^{*}}{\partial \zeta}
\end{aligned}
$$


where $\widetilde{(.)^{*}}$ indicates a Favre filtered quantity evaluated on the CMC grid [5052]. The interaction of the combustion model with the solver used to give the conserved variables is completely different in this compressible solver than in previous CMC implementations. In usual incompressible or low Mach implementations the combustion model provides the mixture density to the fluid solver, whereas in compressible flow the mixture density is governed by the continuity equation. The CMC model is coupled to the Navier Stokes equations through the mass source term in the equation for the evolution of mass fraction of $\mathrm{O}_{2}$ and the energy source term due to formation enthalpies of the species produced or consumed.

The following subsections detail the determination of the modelled terms, and the interaction between the three-dimensional CFD grid and the CMC grid. In this paper the CMC equations are solved in one, two and three spatial dimensions on a reduced grid to allow maximum computational resolution for the turbulent flow field.

An important point to emphasise is that throughout this paper several modelling assumptions are employed from the current state-of-the-art in nonpremixed flows. These are not expected to be optimal for the premixed problem. This is particularly true for the conditional scalar dissipation rate where the required modeling is expected to be different for premixed combustion. However, borrowing from advances in non-premixed flames provides a useful starting point for further model development and to demonstrate the utility of the proposed numerical algorithm.

\subsubsection{Filtered Density Function}

There are several potential forms which can be employed for the Filtered Density Function (FDF). A recent paper by Floyd et al. [53] compared the typical Beta function with the top-hat distribution, where the top-hat assumes a linear distribution of progress variable within each cell. They concluded that the top-hat FDF was superior for use in time dependent turbulent flows. This filter has advantages in the simplicity of implementation, hence is used here.

As detailed by Floyd et al. [53], in LES, the Filtered Density Function (FDF) represents 'the states in the vicinity of a point (within one filter width) for one realisation at one single instant in time'. At the filter width the flow should be resolved, assuming that all large scale mixing occurs over a distance equal to the integral width $\ell$, where $\ell>>\Delta$ and $\Delta$ is the filter width.

The FDF is constructed as a function of the Favre filtered progress vari- 
able $\tilde{c}$ calculated from the advection of $\mathrm{O}_{2}$. The mass fraction of $\mathrm{O}_{2}$ is chosen as progress variable as it is close to the mean molecular mass of the mixture, and tracks the reaction progress well. An 'a-priori' analysis conducted by Hawkes et al. [27] also demonstrated that the first order CMC assumption holds reasonably well for the major species for a lean premixed methane jet. The variance of the progress variable requried to construct the top hat is estimated using a gradient model $[54,55]$ computed via a discretisation suggested by Floyd et al. [53],

$$
\tilde{c^{\prime 2}}=\frac{C_{c^{\prime}}}{4}\left[\left(\tilde{c_{n}}-\tilde{c_{s}}\right)^{2}+\left(\tilde{c_{e}}-\tilde{c_{w}}\right)^{2}+\left(\tilde{c_{u}}-\tilde{c_{d}}\right)^{2}\right]
$$

where the subscripts $n, s, e, w, u$ and $d$ refer to the neighbouring cell locations 'North', 'South', 'East', 'West', 'Up' and 'Down' with respect to the current cell, and $C_{c^{\prime}}=1 / 12$. The progress variable variance is limited to a maximum of $(1-\tilde{c}) \tilde{c}$ as the progress variable must lie between 0 and 1 . This limit also represents the binary mixture limit, where the cell contains only pure unburnt fuel oxidant mixture and pure burnt gas, and is in practise only approached close to the jet inlet. The variance is then used to calculate the FDF, which can take one of five functional forms as outlined by Borghi and Moreau [56], where full details are given in Table 1 of [53]. A slightly modified approach is used here, where if the whole of the top hat function lies in one conditional bin the FDF is spread over two cells such that the filtered progress variable is conserved.

Using this approximation, the combination of scalar variance and turbulent diffusivity correctly predicts the location of the peak conditional scalar dissipation in comparison to experimental results for a turbulent Bunsen flame [57, 58]. The FDF for the CMC grid is then computed as a density weighted average of all of the FDF's in the CFD cells within the CMC cell,

$$
\tilde{P}^{*}(\zeta)=\frac{\int_{V_{C M C}} \bar{\rho} \tilde{P}(\zeta) d V^{\prime}}{\int_{V_{C M C}} \bar{\rho} d V^{\prime}} .
$$

Quantities required on the CMC grid can then be computed by integrating over all CFD cells within a single CMC cell, i.e. for a function $f \mid \zeta$

$$
\widetilde{f * \mid \zeta}=\frac{\int_{V_{C M C}} \bar{\rho} \widetilde{f \mid \zeta} \tilde{P}(\zeta) d V^{\prime}}{\int_{V_{C M C}} \bar{\rho} \tilde{P}(\zeta) d V^{\prime}}
$$


It is worth noting that this adds an additional modelling uncertainty in the quality of the conditionally filtered values gained by integrating over a CMC cell as the CMC cell must contain sufficient CFD cells to give a good approximation to the actual conditional mean.

\subsubsection{CMC Reaction Rates and Conditional Thermodynamic Quantities}

The reaction rates are closed using the first order CMC assumptions,

$$
\overline{\dot{w}}_{k} \mid \zeta=\frac{\overline{\dot{W}_{k}\left(Q^{*}\left|\zeta, \tilde{T}^{*}\right| \zeta, \bar{p}^{*} \mid \zeta\right)} \mid \zeta}{\overline{\rho \mid \zeta}}
$$

where $\dot{W}_{k}$ is the conditionally filtered chemical mass production rate. The conventional filtered chemical source terms required in the filtered progress variable and energy equations are recovered using the Favre FDF as follows $[51,52]$ :

$$
\overline{\rho \dot{w}_{k}}=\bar{\rho} \tilde{\dot{w}}_{k}=\int_{0}^{1} \overline{\dot{w}_{k} \mid \zeta} \overline{\overline{\rho \mid \zeta}} P \tilde{(\zeta} \zeta d \zeta
$$

Conditionally filtered thermodynamic quantities are thus required to compute the conditional reaction rates. For an adequately resolved LES it is reasonable to assume pressure equilibrium within a given computational cell i.e. $\bar{p} \mid \zeta=\bar{p}$ within a single cell for moderate Mach numbers. The conditional pressures are calculated as follows

$$
\overline{p^{*} \mid \zeta}=\frac{\int_{V_{C M C}} \bar{p} \tilde{P}(\zeta) d V^{\prime}}{\int_{V_{C M C}} \tilde{P}(\zeta) d V^{\prime}} .
$$

Unlike pressure, the temperature and density will vary significantly if the flame is on the subgrid level. As the reaction rates are typically very sensitive to the conditional temperature distribution, the conditionally filtered temperatures are computed by firstly calculating the standardised enthalpy in each CFD cell defined as

$$
\tilde{h}=\sum_{k=1}^{N} \tilde{Y}_{k}\left(\Delta h_{f, k}^{\circ}+\int_{T_{0}}^{\tilde{T}} C_{p} d T\right),
$$

where the temperature is already known within a given CFD cell from the energy equation and species composition. The mean standardised enthalpy is then averaged over the $\mathrm{CMC}$ cell as follows: 


$$
\widetilde{h^{*} \mid \zeta}=\frac{\int_{V_{C M C}} \bar{\rho} \tilde{h} \tilde{P}(\zeta) d V^{\prime}}{\int_{V_{C M C}} \bar{\rho} \tilde{P}(\zeta) d V^{\prime}}
$$

The conditional temperatures can then be computed from the mean conditional standardised enthalpy at each conditional bin and $Q *$. This approach has been used successfully in RANS simulations by Kent [59], and permits the influence of temperature inhomogeneities due to compressible flow features on the reaction rates where the gradients caused by compressibility are reasonably represented by the CMC grid.

The conditional densities are required to calculate $\widetilde{w_{k}^{*} \mid \zeta}$, and these are computed from the conditional pressures and temperatures through the ideal gas equation of state, i.e. $\overline{\rho \mid \zeta}=\overline{p|\zeta M| \zeta} / \widehat{R T \mid \zeta}$, where $M$ is the mixture molecular weight and $R$ the universal gas constant.

\subsubsection{Conditional Scalar Dissipation}

There are several possible models which can be applied for scalar dissipation in non-premixed flames [60-64]. As the turbulent diffusivity must be modelled to close the dissipation terms in physical space for the conditionally filtered mass fractions, it is also employed to compute the scalar dissipation,

$$
\tilde{N}=\overline{D_{t}} \frac{\tilde{c^{\prime 2}}}{C_{c^{\prime}} \Delta^{2}} .
$$

where it has been assumed that the relationship $\tilde{c}^{2}=C_{c^{\prime}} \Delta^{2}(\nabla \tilde{c})^{2}$ employed in non-premixed [53] combustion is also valid for premixed. For high Damköhler number flames this will not be physically correct, however the DNS simulated here is at a relatively low Damköhler number which permits this coarse approximation. In addition, it is employed here to demonstrate the stability and capability of the proposed numerical method. The turbulent diffusivity $\overline{D_{t}}$ is approximated using the eddy diffusivity approximation,

$$
\overline{D_{t}}=\frac{\bar{\nu}_{t}}{S c_{t}}=\frac{\left(C_{S} \Delta\right)^{2}}{S c_{t}} \sqrt{2 S_{i j} S_{i j}},
$$

with the turbulent Schmidt number $S c_{t}=0.4$, and the progress variable variance $\tilde{c^{\prime 2}}$ is computed using Eqn. (17). To gain $\widetilde{N^{*} \mid \zeta}$ the values of $\tilde{N}$ computed on the CFD grid are conditionally filtered using the density weighted $\mathrm{FDF}$, i.e. 


$$
\widetilde{N^{*} \mid \zeta}=\frac{\int_{V_{C M C}} \bar{\rho} \tilde{N} \tilde{P}(\zeta) d V^{\prime}}{\int_{V_{C M C}} \bar{\rho} \tilde{P}(\zeta) d V^{\prime}}
$$

\subsubsection{Conditional Velocities}

The conditional velocities are required to calculate the convective flux of conditional mass fractions at a cell interface. Following previous work [13, 17 it is assumed that the conditional velocities are independent of progress variable for each cell interface, i.e. $\widetilde{u_{i} \mid \zeta}=\widetilde{u}_{i}$ in each CFD cell. This model is yet to be validated against experimental data, and is equivalent to assuming that the fluctuations around the conditional filtered velocities at the subgrid level are well represented by the fluctuations resolved on the grid scale. This may be reasonable for numerically well resolved flames, however may not be accurate at higher Damköhler number. Despite this caveat, the previously mentioned LES studies gain very good results using this approximation.

Computationally the conditional velocities are required at the $\mathrm{CMC}$ cell interface, thus $\widetilde{u_{i} \mid \zeta}=\tilde{u}_{i}^{R S}$ where $\tilde{u}_{i}^{R S}$ is computed from the solution of the Riemann problem at each CMC cell interface. The conditional velocities are then gained by integrating over the CMC cell boundaries using Eqn. (19), substituting $f=\tilde{u}_{i}^{R S}$. This ensures consistency as the CMC and CFD grid converge.

\subsubsection{Reaction Models}

The reaction model employed in this work is a reduced model for lean methane-air combustion developed specifically for a lean premixed slot flame conducted by Sankaran et al. [26]. The mechanism used has 72 elementary reactions with 17 species, 4 of which are steady state, hence requiring the computation of 13 species.

\subsubsection{Transfer of Quantities to the CFD Grid}

The CFD grid requires knowledge of the mixture composition in order to calculate local temperatures, pressures, mixture specific heat capacities and transport properties. In addition, the mass production rate of $\mathrm{O}_{2}$ and the change in formation enthalpies must be calculated. The mixture composition is computed following the standard transform

$$
\tilde{Y}=\int_{0}^{1} Q^{*} \tilde{P}(\zeta) d \zeta
$$


The temperatures are computed using Newton-Raphson iteration from the known mixture composition and density plus polynomial $\mathrm{C}_{p}(\tilde{T})$, and pressures from the ideal gas law. All transport properties can be calculated easily once the temperature has been determined.

\section{Numerical Discretisation}

The key advantage of using a finite volume compressible method is that all of the terms in the governing equations can be integrated directly without need for costly implicit iterative loops. This makes the codes easier to parallelise and more easily applied to complex geometries.

This section presents a finite volume discretisation of the governing equations in both physical and progress variable space, allowing a simple yet robust numerical solution.

\subsection{Navier Stokes Equations}

The compressible Navier Stokes equations (continuity, momenta, energy and a single equation for mass fraction of $\mathrm{O}_{2}$ ) are solved using a fifth order in space, second order in time finite volume Godunov method in a newly developed code 'CHOC' (Compressible High-Order Combustion). This employs the modified fifth order accurate reconstruction method of Thornber et al. [36], in conjunction with the HLLC Riemann solver of Toro [65].

The solution to the Riemann problem requires the use of reconstructed thermodynamics quantities such as pressure, the speed of sound, and ratio of specific gases. The values of pressure, density and the mixture ratio of specific heats $\gamma=C_{p} / C_{v}$ are calculated on the CFD grid using knowledge of species mass fractions from the $\mathrm{CMC}$ grid, and then reconstructed to the cell interface using the same fifth order reconstruction as employed for the conserved variables. This avoids having to do a costly Newton-Raphson iteration at each cell interface to compute the pressure or temperature based on the reconstructed material properties, and has negligible influence on the accuracy of the solution to the Riemann problem. The species diffusion, viscous terms and heat diffusion are discretized using second order central differences.

\section{2. $C M C$ Equations}

In a contrast to previous discretisations where ODE solvers have been employed in the solution of the CMC equations, the approach taken here is 
to discretise as a finite volume in physical space, conditional space and time. This is possible as the time step is much smaller in compressible CFD codes due to the CFL criteria. A key numerical difficulty in the solution of the $\mathrm{CMC}$ equations is their non-conservative nature.

As the equations are solved using the method of lines, this section will outline the numerical method for solving the one dimensional system of equations, where the extension to three dimensions is straightforward. The inviscid, non-reacting component of the non-conservative CMC equation gives

$$
\frac{\partial Q^{*}}{\partial t}+\widetilde{u \mid \zeta} \frac{\partial Q^{*}}{\partial x}=0
$$

which is rewritten as a conservative equation minus a correction term,

$$
\frac{\partial Q^{*}}{\partial t}+\frac{\partial \widetilde{u^{*} \mid \zeta} Q^{*}}{\partial x}-Q^{*} \frac{\partial \widetilde{u^{*} \mid \zeta}}{\partial x}=0 .
$$

Following the discretisation proposed by Abgrall [66], the cell interface flux $F^{i+1 / 2}$ and $F^{i-1 / 2}$ in physical space for cell $i$ is computed as

$$
\begin{aligned}
& F^{i+1 / 2}=\left(Q^{*} \widetilde{u^{*} \mid \zeta}\right)^{R S, i+1 / 2}-Q^{*, k} \widetilde{u \mid \zeta}{ }^{*, R S, i+1 / 2} \\
& F^{i-1 / 2}=\left(Q^{*} \widetilde{u^{*} \mid \zeta}\right)^{R S, i-1 / 2}-Q^{*, k} \widetilde{u \mid \zeta}^{*, R S, i-1 / 2} .
\end{aligned}
$$

where $(.)^{R S}$ indicates a term arising from the solution of the Riemann problem at the cell interface using fifth order accurate reconstruction.

The final non-conservative flux term on the right hand side of Eqn. (16) is also discretised in the same manner but in progress variable space. The effective velocity in progress variable space $\widetilde{S_{c}^{*} \mid \zeta}$ is first calculated using the knowledge that the mass fraction of $\mathrm{O}_{2}$ is related linearly to the progress variable and never changes, giving

$$
\widetilde{\dot{w}_{O_{2}}^{*} \mid \zeta}=\widetilde{S_{c}^{*} \mid \zeta} \frac{\partial Q_{O_{2}}^{*}}{\partial \zeta}
$$

where $\partial Q_{\mathrm{O}_{2}}^{*} / \partial \zeta=Y_{\mathrm{O}_{2}}^{b}-Y_{\mathrm{O}_{2}}^{u}$ where $u$ and $b$ denote burnt and unburnt mass fractions. Computing the reaction rate $\widetilde{\dot{w}_{\mathrm{O}_{2}}^{*} \mid \zeta}$ then the velocity in mixture fraction space can be computed at the cell centres as follows

$$
\widetilde{S_{c}^{*} \mid \zeta}=\frac{\widetilde{w_{O_{2}}^{*} \mid \zeta}}{\partial Q_{O_{2}}^{*} / \partial \zeta}=\frac{\widetilde{\dot{w}_{O_{2}}^{*} \mid \zeta}}{Y_{O_{2}}^{b}-Y_{O_{2}}^{u}},
$$


which is then reconstructed at fifth order accuracy to the cell interface. This forms a Riemann problem at the cell interfaces where the upwind direction is chosen based on the average of the left and right progress variable space velocities. Due to the interpolation process and selection of the upwind direction, the resultant fluxes may no longer satisfy Equation (33). To correct for this, $\widetilde{S_{c}^{*} \mid \zeta}$ is linearly rescaled within each cell to ensure that the advected CMC equation for $\mathrm{O}_{2}$ remains in synchrony with the conditional coordinates. The resultant value of velocity in mixture fraction space is used as the advection velocity in progress variable space for all conditional mass fractions in the CMC equations.

The diffusive terms in conditional and physical space are discretised in exactly the same manner as the diffusive terms in the Navier Stokes equations using second order central differences. To maintain consistency between the progress variable and conditional mass fraction of $\mathrm{O}_{2}$ it is assumed that there is zero net flux through the first and last cell interface (i.e. from $\tilde{c}<0$ and $\tilde{c}>1$ ) due to scalar dissipation.

\subsection{Time Discretisation}

As explicit time integration is proposed, there are several potential restrictions on the time step size. The Euler equations impose the criteria $C F L=(\Delta t(|\tilde{u}|+a)) / \Delta<1$, and the conditional velocity requires $\Delta t\left(\widetilde{u_{i}^{*} \mid \zeta}\right) / \Delta<1$ which is less restrictive than the first condition. Fluxes in conditional space offer two further restrictions, firstly due to the effective velocity in conditional space giving $\Delta t \widetilde{S_{c}^{*} \mid \zeta} / \Delta \zeta<1$, and a Péclet number restriction due to the conditional scalar dissipation rate $2 \Delta t \widetilde{N^{*} \mid \zeta} / \Delta \zeta^{2}<1$. In the current validation cases the final criteria (due to $\widetilde{N^{*} \mid \zeta}$ ) dominates on all grids. The stable time step is not limited by that required to accurately represent the chemical reaction rate in any case simulated here.

As the time step size is thus very small compared to incompressible solvers, there is very little difference in solution when employing second or higher order accurate methods in time with the fifth order reconstruction in space [67]. Hence time integration is achieved using a two stage second order TVD Runge-Kutta method [68]: 


$$
\begin{array}{r}
U^{(1)}=U^{n}+\frac{\Delta t}{\Delta x} f\left(U^{n}\right) \\
U^{n+1}=\frac{1}{2}\left[U^{n}+U^{(1)}+\frac{\Delta t}{\Delta x} f\left(U^{(1)}\right)\right]
\end{array}
$$

As the second order method is more computationally efficient, it is employed here. To improve computational efficiency a second order accurate time split method is employed (also known as fractional time stepping) [69], where the conditional scalar dissipation term (second term on the RHS of Equation. (16) is solved using a time step size dictated by the above Péclet number limit. The remaining terms in the LES-CMC equation and filtered Navier-Stokes equations are evolved over a time step limited by the CFL criteria. The solution vector $U^{n+1}$ is thus constructed as follows:

$$
U^{n+1}=S^{\Delta t / 2} C^{\Delta t} S^{\Delta t / 2}\left(U^{n}\right)
$$

where $S$ and $C$ are solution operators which employ the explicit second order TVD Runge-Kutta method. Simulations run with and without this time splitting show negigible difference between the two solutions.

Both the CMC and the CFD grid have been parallelised very simply in the axial direction only. An interesting point to note is that there is negligible computational effort in the solution of the CMC equations, the majority of the computational time is used to generate the conditionally filtered quantities on the CMC grid from the CFD grid, and recovering the mass production terms in physical space, mixture and thermodynamic properties on the CFD grid (not including calculating the reactions). These processes account for $60 \%$ of the total computational time.

\section{Turbulent Premixed Methane Slot Flame}

The chosen validation case is a comparison to the recent DNS of a lean premixed methane bunsen burner of Sankaran et al. [26]. Case ' $\mathrm{C}$ ' is run here, with a Reynolds number of 2100 based on slot width, a turbulent Reynolds number $\mathrm{Re}_{t}=250$ and a Karlovitz number $K a=225$. The incoming flow is designed such that there is a significant preheat zone hence it is of relevance to the understanding of much higher Reynolds number flows in industrial configurations. 
The premixed fuel/air inlet has an equivalence ratio of 0.7 , and is introduced into the domain at a temperature of $800 \mathrm{~K}$ and mean inflow velocity of $100 \mathrm{~m} / \mathrm{s}$ (rms turbulent fluctuations $\approx 35 \%$ of the mean inflow velocity) with a slot width of $1.8 \mathrm{~mm}$. The laminar coflow velocity is $25 \mathrm{~m} / \mathrm{s}$.

The advantage of employing this dataset for validation is that the uncertainty over the influence of the inlet boundary conditions and reaction scheme are considerably reduced as the same inflow velocity field and chemical mechanism are employed in the current LES as in the DNS. Simulations on the coarsest grid took 8 hours on eight Intel Woodcrest $3 \mathrm{GHz}$ processors, the finest simulation taking two days on sixty-four processors.

\subsection{Numerical Details}

In the following simulations a three dimensional CFD grid is employed along with a reduced dimension CMC grid. The CFD grids from coarse to fine have $75 \times 25 \times 144,100 \times 32 \times 192$ and $150 \times 50 \times 288$ and $200 \times 64 \times 384$ points, where $\mathrm{x}$ is the cross-stream coordinate, $\mathrm{y}$ the homogeneous direction, and $\mathrm{z}$ the axial direction. The computational domain is Cartesian with dimensions $x \times y \times z=25 \mathrm{~mm} \times 3.6 \mathrm{~mm} \times 24 \mathrm{~mm}$. The CMC grid was discretised with 100 points in progress variable space and has one point to each CFD point in the axial direction. The grid is uniform in the $y$ and $z$ directions, however exponential clustering is employed in the $x$ direction such that there is a homogeneous grid containing $60 \%$ of the points lie in the range $-4 \mathrm{~mm} \leq x \leq$ $4 \mathrm{~mm}$ where the grid is nearly isotropic.

The baseline simulations have all been run with a purely axial CMC grid, as it is believed that the downstream transport of conditional moment was of prime importance. This permits averages over the cross-stream direction thus increasing the number of CFD points in each conditional bin which improves the quality of the conditional filtered values. Two additional simulations are presented using CMC grids of $15 \times 1 \times 320$ and $15 \times 5 \times 320$ with a CFD grid size of $150 \times 50 \times 320$. The grid dimension in the axial direction was chosen to permit and even number of points per processor when running on 64 processors. As the integral length scale is on the order of $1.2 \mathrm{~mm}$, the three dimensional grid has at least one CMC cell for each integral length in the $x$ and $y$ directions.

The boundary conditions employed in the axial direction are a simple extrapolated outlet, and the inlet reads in a prescribed turbulent flow field which is identical to that specified for the DNS. Temperature and internal energy are fixed at the inlet, whereas the FDF and turbulent diffusivities are 
extrapolated. The cross-stream direction boundary conditions are outflows where all quantities are extrapolated. The extent of the domain in the crossstream direction has been varied until the dynamics of the slot burner are grid-independent. Finally, periodic boundary conditions are enforced in the homogeneous direction.

Results have been time averaged using 100 instantaneous outputs over a period of $1 \mathrm{~ms}$ following two initial flow through periods to allow the flow to become stabilised (based on a flow through time of $0.24 \mathrm{~ms}$ ).

\subsection{Results}

Figure 1 shows instantaneous flow visualisation of $|\nabla \tilde{c}|$ on the finest grid, and three dimensional isosurfaces of $\tilde{c}=0.65$. An instantaneous visualisation of gradients of the Favre filtered progress variable is shown in Figure 1 for the highest resolution grid. The instantaneous flame structure is physically correct, with a clear preheat zone at intermediate progress variables due to turbulent mixing, and steep gradients in the with the heat release zone.

Statistical comparisons are now conducted based on ensemble averages of the LES and DNS data. The ensemble is formed by averaging in time, over the homogeneous y direction and exploiting symmetry about the jet axis. The ensemble-average LES results are compared directly to ensemble-averaged DNS results since filtering prior to ensemble averaging has little effect on the quantities investigated. Comparisons of contour lines of progress variable for each grid level of the LES with the DNS study are shown in Figure 2. When compared to the DNS results, the initial turbulent mixing is very well represented, up to $\tilde{c} \approx 0.4$, and there is excellent agreement for the width of the flame predicted in LES compared to DNS.

The figure suggests that the reaction rates are too low in the core, leading to an overestimation of flame length $\left(20.5 \mathrm{~mm}\right.$ c.f. $\left.\mathrm{L}_{f}=18.5 \mathrm{~mm}\right)$. Within the core of the jet the mean flow rate is very high (on the order of $100 \mathrm{~m} / \mathrm{s}$, fluctuating velocities up to $\approx 200 \mathrm{~m} / \mathrm{s}$ ), hence any inaccuracies in turbulence and combustion modelling will be magnified due to strong turbulence-chemistry interaction. This is most noticable for $0.4<\tilde{c}<0.5$ where the contour lines in the DNS are closer together than the LES results at all grid resolutions.

There is surprisingly little variation in the results going from the coarsest to the finest grid level in the LES - despite the fact that the finest grid has 20 times the points of the coarsest. This is very promising as it indicates that a consistent LES solution can be gained with a modest grid resolution (this may not apply in cases with higher flow velocities closer to extinction). 
Figures 3 shows the progress variable contours for the two dimensional and three dimensional CMC grid compared to the DNS result. There is no improvement over the one dimensional CMC grid for this case, where it appears that an improvement in resolution in physical space is counterbalanced by the reduction in accuracy of the conditionally filtered values (or the errors in the modelling assumptions). This is further confirmed in Figure 4 which shows an instantaneous visualisation at $0.5 \mathrm{~ms}$ of the divergence of the progress variable for the different dimensionality of CMC grid, where very little difference can be seen between the three simulations.

Cross-stream quantitative data have been extracted for the one-dimensional CMC simulations along $z=0.005 \mathrm{~m}\left(z / L_{f} \approx 25 \%\right)$ and $z=0.015 \mathrm{~m}\left(z / L_{f} \approx\right.$ $80 \%)$. Figures 5 and 6 show the mean density, temperature and velocities along each of these lines. Comparisons of density and temperature show excellent agreement with DNS, with a maximum error of $9 \%$ in density and $3 \%$ in temperature in the core at the downstream position.

The mean axial velocity $(\tilde{w})$ match the DNS data, however at the downstream position the mixing layer is too narrow. This is likely a result of the underprediction of the reaction rates. Moderate agreement is gained for the cross-stream velocity component $\tilde{u}$, however it should be noted that the mean cross-stream velocity is of the order of $5 \%$ of the mean freestream velocity. Given the imposed high level of turbulent inflow fluctuations, where the instantaneous values of cross-stream velocity are on the order of $\pm 80 \mathrm{~m} / \mathrm{s}$, these mean velocities are difficult to converge. In particular, the $\tilde{u}$ velocity in the farfield shows some sensitivity to grid resolution and the exact description of the cross-stream boundary conditions.

The species mass fractions of $\mathrm{CH}_{4}, \mathrm{O}_{2}, \mathrm{H}_{2} \mathrm{O}, \mathrm{CO}_{2}, \mathrm{OH}$ and $\mathrm{CO}$ are plotted at in Figure 7 and 8. The mass fractions of $\mathrm{CH}_{4}, \mathrm{O}_{2}, \mathrm{H}_{2} \mathrm{O}$, and $\mathrm{CO}_{2}$ are in very good agreement with DNS, with a small overestimation of $\mathrm{CO}_{2}$ and $\mathrm{H}_{2} \mathrm{O}$ on the boundary of the flame. $\mathrm{CO}$ is underestimated by $\approx 19 \%$, but matches the DNS data qualitatively. A comparison of the mass fraction of $\mathrm{OH}$ show that there is a significant difference in form at $z=0.005 \mathrm{~m}$. There is a peak in the DNS data at $x \approx 0.0026 \mathrm{~m}$ which is not present in the LES data at any grid resolution. At $z=0.015 \mathrm{~m}$ the match with DNS is qualitatively good with an underestimation of $\mathrm{OH}$ levels by $24 \%$. Using two or three physical dimensions for the CMC model improves the agreement by approximately $5 \%$ compared to the one dimensional CMC model at $z=0.015 \mathrm{~m}$, with a marginally larger peak at $z=0.005 \mathrm{~m}$.

A comparison of conditional mass fractions of $\mathrm{CH}_{4}, \mathrm{OH}$ and $\mathrm{CO}$ can be 
seen in Figure 9, where the DNS data has been averaged into 40 conditional bins. The DNS values were conditionally filtered on the local progress instantaneous variable defined with $\mathrm{O}_{2}$ mass fraction, by averaging in the homogeneous direction and in time. The transverse dependence of conditional values is of minor importance and was ignored. The LES match very well the DNS data both qualitatively and quantitatively. Initially very high levels of conditional scalar dissipation lead a rapid decrease in gradients in conditional space. It appears that the modelled conditional scalar dissipation rate in the LES overestimates that present in the actual DNS, or that the conditional reaction rates close to the jet inlet are lower. This is particularly clear in the $\mathrm{OH}$ and $\mathrm{CO}$ conditional mass fractions. This lead to lower conditional mass fractions in the downstream part of the flame, explaining the discrepancies in the prediction of $\tilde{Y}_{O H}$ in Figure 8 .

After $z=0.003 \mathrm{~m}$ the scalar dissipation decreases and chemical reactions begin to counter-balance the conditional dissipation. The decrease in conditional scalar dissipation rate is caused jointly by a reduction in conditional filtered progress variable divergence (as seen in [57]), and a decrease in turbulent diffusivity with axial distance. Overall, peak conditional $\mathrm{OH}$ and $\mathrm{CO}$ levels are underestimated by $10 \%$ in the LES compared to DNS. The location of the peak of the conditional mass fraction and the variation of this location in the downstream direction is very well predicted in the LES.

\section{Conclusions}

This paper has summarised the design of a novel algorithm for fully compressible Large-Eddy Simulation of premixed combustion problems employing the Conditional Moment Closure model. This new algorithm has been applied to a lean premixed slot bunsen burner, and results compared to DNS, demonstrating the first integration of a CMC subgrid model into a fully compressible unsteady algorithm, and first application to an LES of a premixed flame.

It has shown that a fully explicit very high order compressible finite volume method coupled with CMC is computationally efficient and easily parallelised. This is a substantial variation from existing state-of-the-art algorithms. The algorithm has a wide range of potential applications in industrial gas turbine combustion acoustic instabilities, industrial safety problems, homogeneous charge compression ignition engines and high speed propulsion. 
Clearly there are many improvements which can be pursued, most notably improvements to the modelling of the conditional scalar dissipation rate, the FDF and the turbulent diffusivity. Given the fundamentally different behaviour of non-premixed and premixed turbulence, the current modelling choices (based primarily on existing methods for non-premixed flows) are not correct on physical grounds in higher Damköhler number combustion regimes, however good agreement with the present, low Damköhler number DNS data is achieved with these coarse assumptions. The chosen test case has clearly demonstrated that the proposed algorithm is capable of stably simulating a fully compressible premixed problem in a computationally efficient manner.

\section{Acknowledgements}

The author would like to acknowledge the advice and discussions with Prof. J.H. Kent which have been invaluable.

This work has been possible due to the support of the International Visiting Research Fellowship program from the University of Sydney, and a Global Research Award from the Royal Academy of Engineering.

Professors Bilger and Masri are supported by the Australian Research Council.

The DNS data were generated using resources of the National Center for Computational Sciences at Oak Ridge National Laboratory (NCCS/ORNL) which is supported by the Office of Science of the DOE under contract no. DE-AC05-00OR22725.

\section{References}

[1] R. Bilger, S. Pope, K. Bray, J. Driscoll, Paradigms in turbulent combustion research, Proc. Combust. Inst. 7 (2005) 21-42.

[2] C. Law, Combustion at a crossroads: Status and prospects, Proc. Combust. Inst. 31 (2007) 1-29. Doi:10.1016/j.proci.2006.08.124.

[3] H. Pitsch, Large-eddy simulation of turbulent combustion, Annu. Rev. Fluid Mech. 38 (2006) 453-482.

[4] S. Pope, Turbulent Flows, Cambridge University Press, 2000. 
[5] P. Sagaut, Large Eddy Simulation for Incompressible Flows, Springer Verlag, 2001.

[6] T. Poinsot, D. Veynante, Theoretical and Numerical Combustion, Edwards, 2005.

[7] S. Pope, Computations of turbulent combustion: progress and challenges, in: Proceedings of the 23rd Symp. (Int.) on Combustion, The Combustion Institute, pp. 591-612.

[8] A. Lipatnikov, J. Chomiak, Effects of premixed flames on turbulent and turbulent scalar transport, Prog. Energ. Combust. 36 (2010) 1-102. Doi:10.1016/j.pecs.2009.07.001.

[9] R. Bilger, Conditional moment closure for turbulent reacting flow, Phys. Fluids A 5 (1993) 436-444.

[10] A. Klimenko, Multicomponent diffusion of various scalars in turbulent flow, Fluid Dyn. 25 (1990) 327-333.

[11] A. Klimenko, R. Bilger, Conditional moment closure for turbulent combustion, Prog. Energ. Combust. 25 (1999) 595-687.

[12] S. Kim, H. Pitsch, Conditional filtering method for large-eddy simulation of turbulent nonpremixed combustion, Phys. Fluids 17 (2005) 105103.

[13] S. Navarro-Martinez, A. Kronenburg, F. Di Mari, Conditional moment closure for large eddy simulations, Flow Turbul. Combust. 75 (2005) 245-274.

[14] S. Navarro-Martinez, A. Kronenburg, LES-CMC simulations of a turbulent bluff-body flame, Proc. Combust. Inst. 31 (2007) 1721-1728.

[15] S. Navarro-Martinez, A. Kronenburg, LES-CMC simulations of a lifted methane flame, Proc. Combust. Inst. 32 (2009) 1509-1516.

[16] A. Triantafyllidis, E. Mastorakos, R. Eggels, Large Eddy Simulations of forced ignition of a non-premixed bluff-body methane flame with conditional moment closure, Combust. Flame 156 (2009) 2328-2345. Doi:10.1016/j.combustflame.2009.05.005. 
[17] A. Triantafyllidis, E. Mastorakos, Implementation issues of the Conditional Moment Closure model in large eddy simulations, Flow Turbul. Combust. 84 (2010) 481-512.

[18] S. Martin, J. Kramlich, G. Kosaly, J. Riley, The premixed conditional moment closure method applied to idealized lean premixed gas turbine combustors, J. Eng. Gas Turb. Power 125 (2003) 895-900.

[19] R. Bilger, Marker fields for turbulent premixed combustion, Combust. Flame 138 (2004) 188-194.

[20] C. Duwig, L. Fuchs, Large eddy simulation of turbulent premixed combustion using a marker field, Combust. Sci. Technol. 179 (2007) 21352152. Doi: 10.1080/00102200701386164.

[21] N. Smith, Development of the Conditional Moment Closure method for modelling turbulent combustion, Ph.D. thesis, University of Sydney, 1994.

[22] C. Fureby, A fractal flame-wrinkling large eddy simulation model for premixed turbulent combustion, Proc. Combust. Inst. 30 (2005) 593601. Doi:10.1016/j.proci.2004.08.068.

[23] H. Weller, G. Tabor, A. Gosman, C. Fureby, Application of a flamewrinkling LES combustion model to a turbulent mixing layer, Proc. Combust. Inst. 27 (1998) 899-907.

[24] M. Kirkpatrick, S. Armfield, A. Masri, S. Ibrahim, Large Eddy Simulation of a propagating turbulent premixed flame, Flow Turbul. Combust. 70 (2003) 1-19.

[25] C. Duwig, C. Fureby, Large eddy simulation of unsteady lean stratified premixed combustion, Combust. Flame 151 (2007) 85-103. Doi: 10.1016/j.combustflame.2007.04.004.

[26] R. Sankaran, E. Hawkes, J. Chen, T. Lu, C. Law, Structure of a spatially developming turbulent lean methane-air bunsen flame, Proc. Combust. Inst. 31 (2007) 1291-1298. Doi:10.1016/j.proci.2006.08.025.

[27] E. Hawkes, R. Sankaran, J. Chen, A DNS database for validation of models for extinction and reignition, in: 4th International Workshop on CMC and unsteady flamelets, Heidelberg, Germany. 
[28] A. Vreman, J. van Oijen, L. de Goey, R. Bastiaans, Subgid scale modelling in Large-Eddy Simulation of turbulent combustion using premixed flamelet chemistry, Flow Turbul. Combust. 82 (2009) 511-535. Doi:10.1007/s10494-008-9159-x.

[29] A. Amsden, KIVA-3V: A block structured KIVA program for engines with vertical or canted valves, Technical Report, Los Alamos, 1997.

[30] O. Colin, F. Ducros, D. Veynante, T. Poinsot, A thickened flame model for large eddy simulations of turbulent premixed combustion, Phys. Fluids 12 (2000) 1843-1863. Doi:10.1063/1.870436.

[31] P. Domingo, L. Vervisch, D. Veynante, Large-eddy simulation of a lifted methane jet flame in a vitiated coflow, Combust. Flame 152 (2008) 415432. Doi: 10.1016/j.combustflame.2007.09.002.

[32] M. Ihme, H. Pitsch, Prediction of extinction and reignition in nonpremixed turbulent flames using a flamelet/progress variable model 2 . application in LES of Sandia flames D and E, Combust. Flame 155 (2008) 90-107. Doi: 10.1016/j.combustflame.2008.04.015.

[33] M. Berglund, E. Fedina, C. Fureby, J. Tegner, Finite rate chemistry Large-Eddy Simulation of self-ignition in a supersonic combustion ramjet, AIAA J. 48 (2010) 540-550. Doi:10.2514/1.43746.

[34] V. Molkov, F. Verbecke, D. Makarov, Les of hydrogen-air deflagrations in a 78.5m tunnel, Combust. Sci. Technol. 180 (2008) 796-808. DOI: 10.1080/00102200801893994.

[35] B. Thornber, D. Drikakis, R. Williams, D. Youngs, On entropy generation and dissipation of kinetic energy in high-resolution shock-capturing schemes, J. Comput. Phys. 227 (2008) 4853-4872. Doi:10.1016/j.jcp.2008.01.035.

[36] B. Thornber, A. Mosedale, D. Drikakis, D. Youngs, R. Williams, An improved reconstruction method for compressible flows with low Mach number features, J. Comput. Phys. 227 (2008) 4873-4894. Doi:10.1016/j.jcp.2008.01.036. 
[37] B. Vreman, A.W. Geurts, H. Kuerten, A priori tests of large eddy simulation of the compressible plane mixing layer, J. Eng. Math. 29 (1995) 299-327.

[38] A. Kolmogorov, A refinement of previous hypotheses concerning the local structure of turbulence in a viscous incompressible fluid at high Reynolds number, J. Fluid Mech. 13 (1962) 82-85.

[39] B. Thornber, A. Mosedale, D. Drikakis, On the Implicit Large Eddy Simulation of homogeneous decaying turbulence, J. Comput. Phys. 226 (2007) 1902-1929. Doi:10.1016/j.jcp.2007.06.030.

[40] E. Garnier, M. Mossi, P. Sagaut, P. Comte, M. Deville, On the use of shock-capturing schemes for large-eddy simulation, J. Comput. Phys. 153 (1999) 273-311. Doi:10.1006/jcph.1999.6268.

[41] F. Grinstein, L. Margolin, W. Rider (Eds.), Implicit Large Eddy Simulation: Computing Turbulent Fluid Dynamics, Cambridge University Press, Cambridge, 2007.

[42] D. Drikakis, Advances in turbulent flow computations using high-resolution methods, Prog. Aerosp. Sci. 39 (2003) 405-424. Doi:10.1016/S0376-0421(03)00075-7.

[43] D. Drikakis, M. Hahn, A. Mosedale, B. Thornber, Large eddy simulation using high-resolution and high-order methods, Phil. Trans. R. Soc. A 367 (2009) 2985-2997. Doi:10.1098/rsta.2008.0312.

[44] B. Thornber, D. Drikakis, Implicit large eddy simulation of a deep cavity using high resolution methods, AIAA J. 46 (2008) 2634-2645. Doi:10.2514/1.36856.

[45] B. Thornber, M. Starr, D. Drikakis, Implicit large eddy simulation of ship airwakes, Aeronaut. J. (2009). Under revision.

[46] B. Thornber, D. Drikakis, D. Youngs, R. Williams, The influence of initial conditions on turbulent mixing due to richtmyer-meshkov instability, J. Fluid Mech. 654 (2010) 99-139. Doi:10.1017/S0022112010000492.

[47] M. Smooke, V. Giovangigli, Formulation of the premixed and nonpremixed test problems, Lect. Notes Phys. 384 (1991) 1-28. 
[48] J. Smagorinsky, General circulation experiments with the primitive equations, Mon. Weather Rev. 91 (1963) 99-164.

[49] A. Burcat, B. Ruscic, Third Millenium Ideal Gas and Condensed Phase Thermochemical Database for Combustion with Updates from Active Thermochemical Tables, Technical Report, Argonne National Laboratory, 2005.

[50] A. Favre, Problems of hydrodynamics and continuum mechanics, statistical equations of turbulent gases, SIAM (1969) 231-266.

[51] R. Bilger, Note on favre averaging in variable density flows, Combust. Sci. Technol. 11 (1975) 215-217.

[52] R. Bilger, Turbulent jet diffusion flames, Progr. Energy Combust. Sci. 1 (1976) 87-109.

[53] J. Floyd, A. Kempf, A. Kronenburg, R. Ram, A simple model for the filtered density function for passive scalar combustion LES, Combust. Theor. Model. 13 (2009) 559-588. Doi:10.1080/13647830802632200.

[54] N. Branley, W. Jones, Large eddy simulation of a turbulent nonpremixed flame, in: Proceedings of the 11th Symposium on Turbulent Shear Flows, Grenoble, France, pp. 21.1-21.6.

[55] F. Mathey, J. Chollet, Large-eddy simulation of turbulent reactive flows, in: Proceedings of the 11th Symposium on Turbulent Shear Flows, Grenoble, France, pp. 16.19-16.24.

[56] R. Borghi, P. Moreau, Turbulent combustion in a premixed flow, Acta Astronaut. 4 (1977) 321-341.

[57] F. O'Young, R. Bilger, Scalar gradient and related quantities in turbulent premixed flames, Combust. Flame 109 (1997) 682-700.

[58] S. Chen, G. Doolen, R. Kraichnan, Z.-S. She, On statistical correlations between velocity increments and locally averaged dissipation in homogeneous turbulence, Phys. Fluids A 5 (1992) 458-463.

[59] J. Kent, Prediction of particulates in turbulent diffusion flames by conditional moment closure, in: Combustion generated fine carbonaceous particles, pp. 605-618. 
[60] E. O'Brien, T.-L. Jiang, The conditional dissipation rate of an initially binary scalar in homogeneous turbulence, Phys. Fluids 3 (1991) 31213123 .

[61] S. Kim, H. Pitsch, Mixing characteristics and structure of a turbulent jet diffusion flame, Phys. Fluids 18 (2006) 075103.

[62] C. Pierce, P. Moin, A dynamic model for subgrid-scale variance and dissipation rate of a conserved scalar, Phys. Fluids 10 (1998) 30413044.

[63] S. Kops, J. Riley, G. Kosaly, A. Cook, Investigation of modeling for nonpremixed turbulent combustion, Flow Turbulence Combust. 60 (1998) 105-122.

[64] S. Girimaji, Y. Zhou, Analysis and modeling of subgrid scalar mixing using numerical data, Phys. Fluids 8 (1996) 1224-1236.

[65] E. Toro, Riemann Solvers and Numerical Methods for Fluid Dynamics, Springer-Verlag, Cambridge, 1997.

[66] R. Abgrall, How to prevent pressure oscillations in multi-component flow calculations: A quasi conservative approach, J. Comput. Phys. 125 (1996) 150-160.

[67] M. Hahn, Implicit large-eddy simulation of low-speed separated flows using high-resolution methods, Ph.D. thesis, Cranfield University, 2008.

[68] C.-W. Shu, Total-variation-diminishing time discretizations, SIAM J. Sci. Stat. Comp. 9 (1988) 1073-1084.

[69] G. Strang, On the construction and comparison of difference schemes, SIAM J. Num. Anal. 5 (1968) 506-517.

[70] H. Steiner, W. Bushe, Large-eddy simulation of a turbulent reacting jet with conditional source estimation, Phys. Fluids 25 (2001) 595-687. 

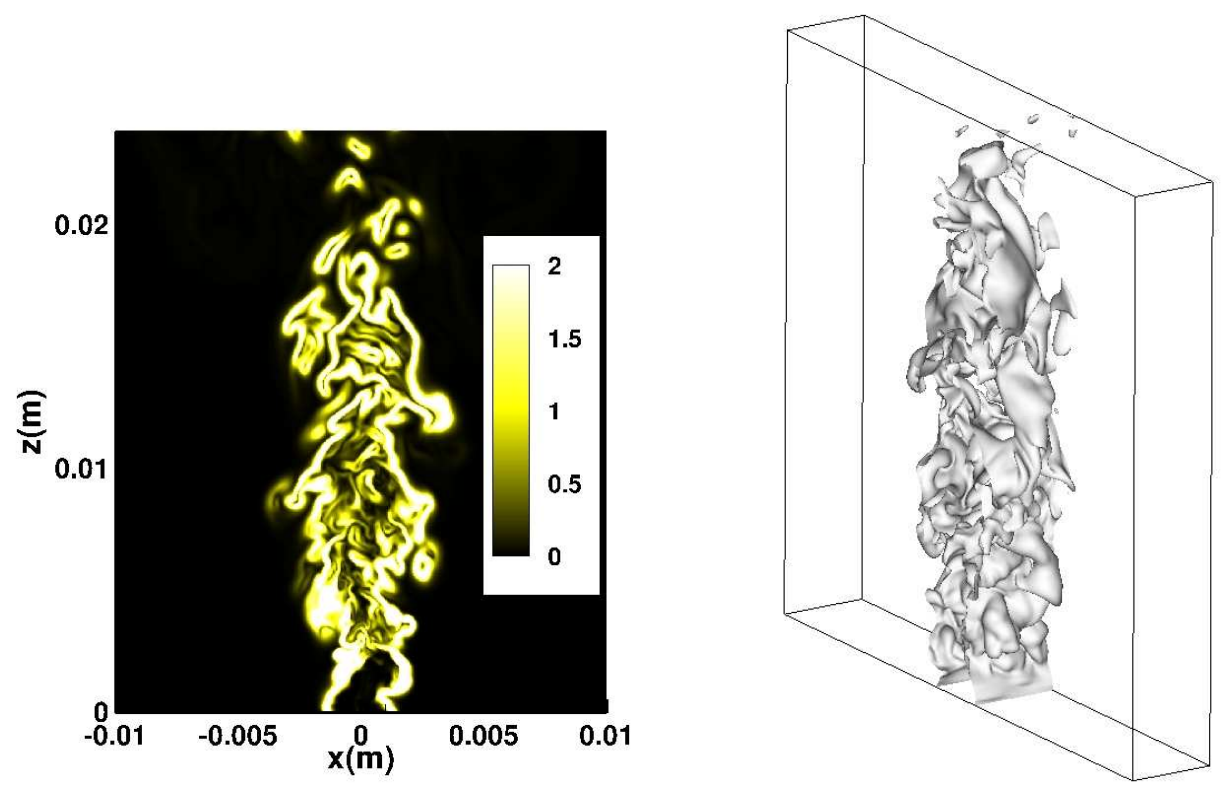

Figure 1:

Figures 


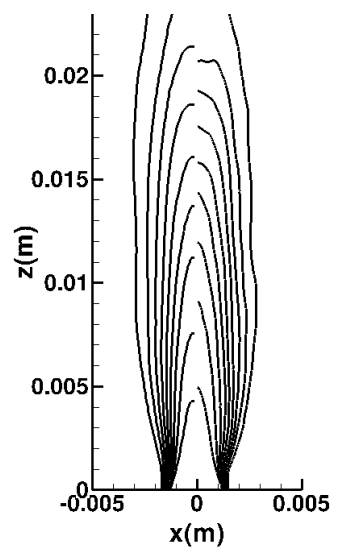

(a) $75 \times 24 \times 144$

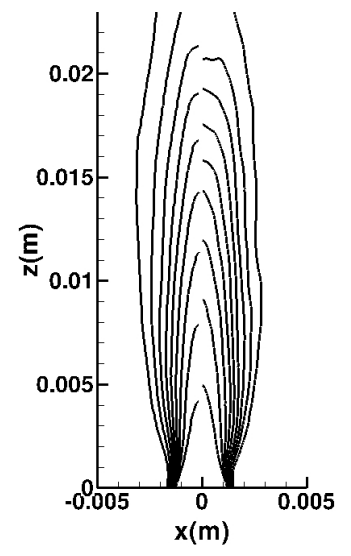

(b) $100 \times 32 \times 192$

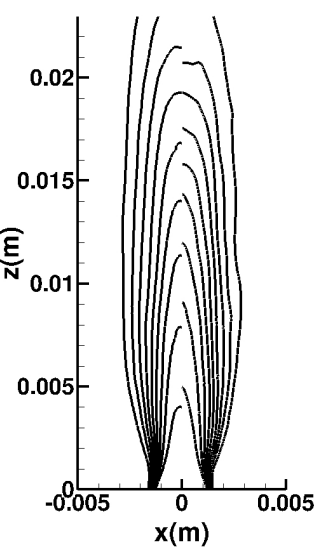

(c) $150 \times 50 \times 288$

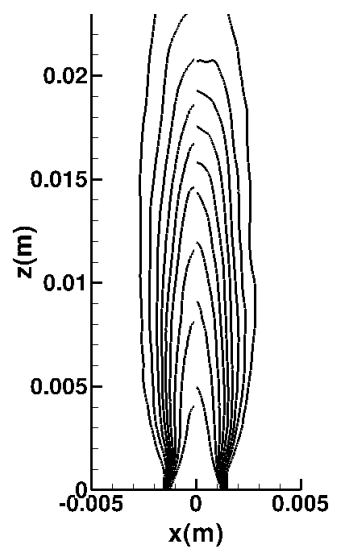

(d) $200 \times 64 \times 384$

Figure 2: 


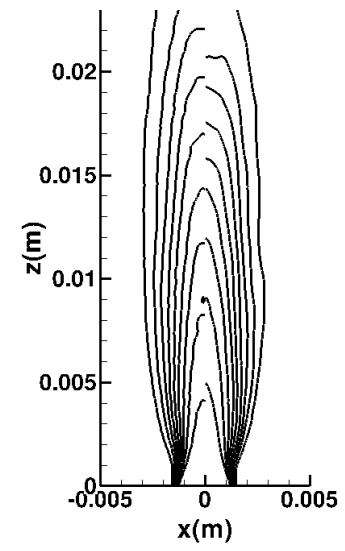

(a) $2 \mathrm{D} \mathrm{CMC}$

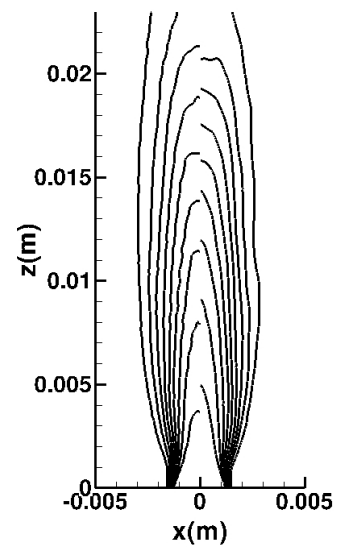

(b) $3 \mathrm{D} \mathrm{CMC}$

Figure 3: 


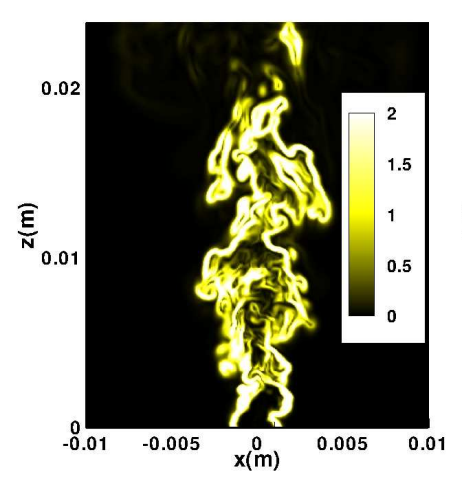

(a) $1 \mathrm{D} \mathrm{CMC}$

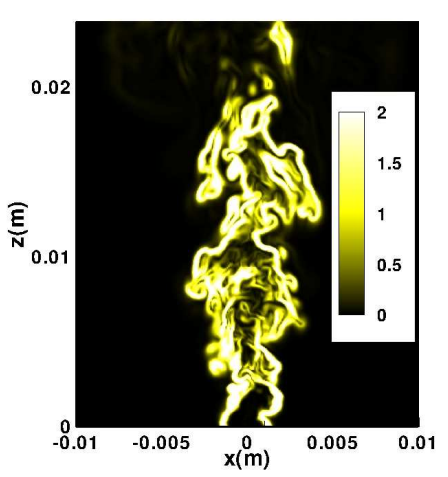

(b) $2 \mathrm{D} \mathrm{CMC}$

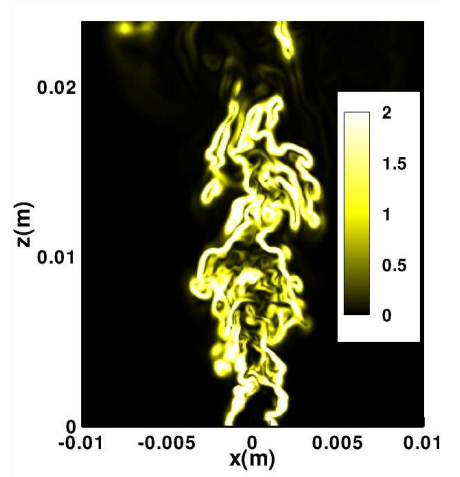

(c) $3 \mathrm{D} \mathrm{CMC}$

Figure 4: 

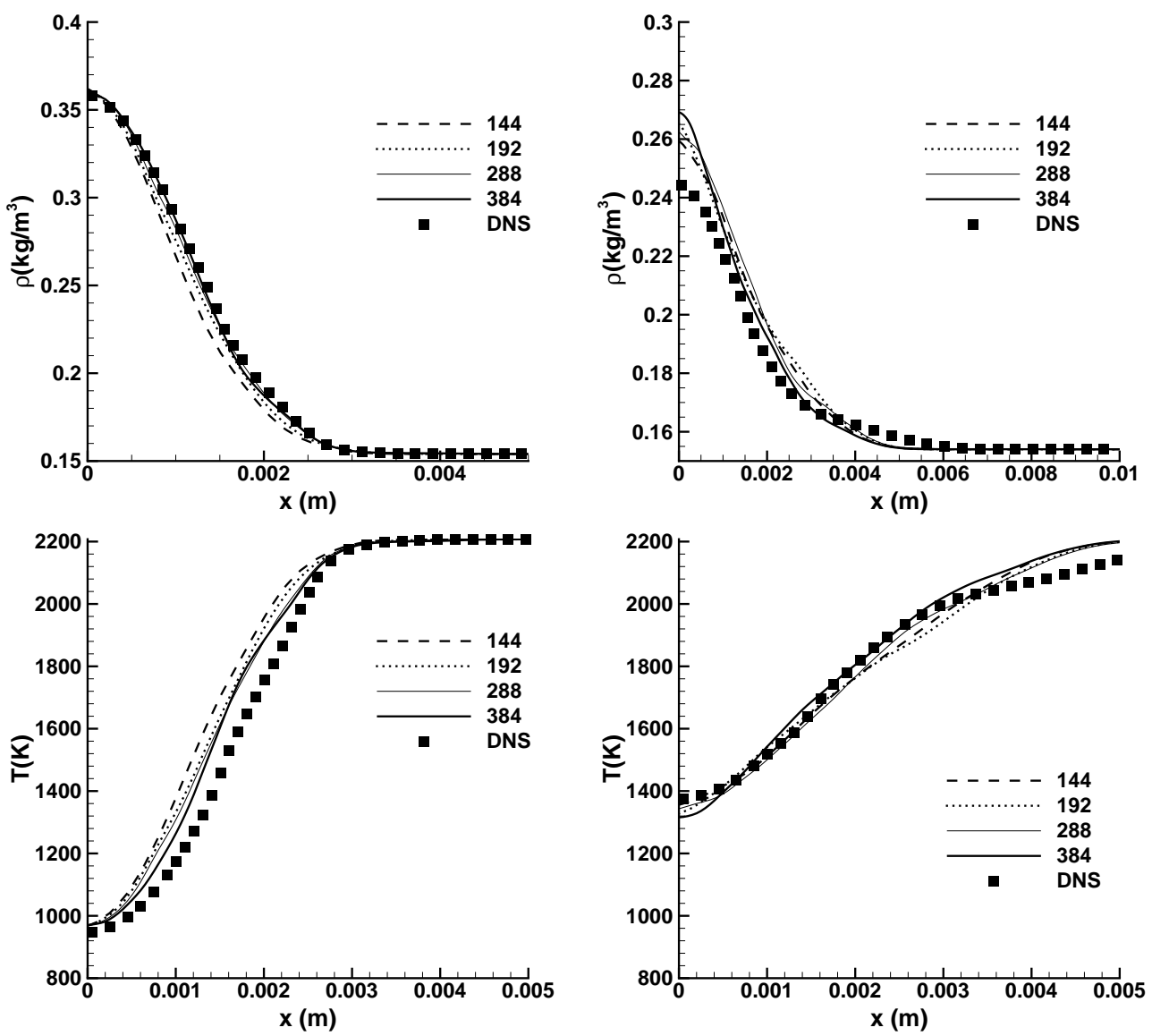

Figure 5: 

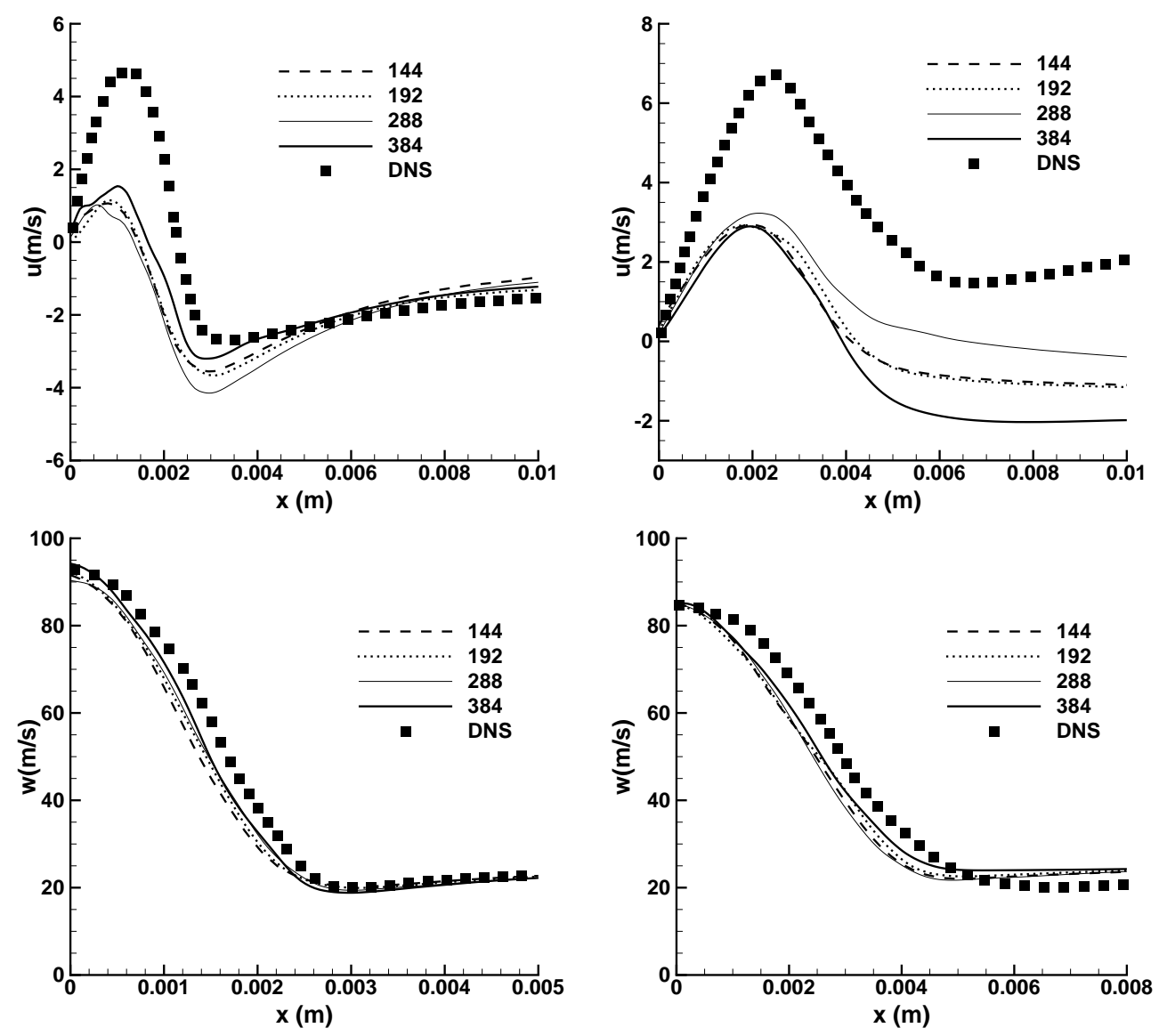

Figure 6: 

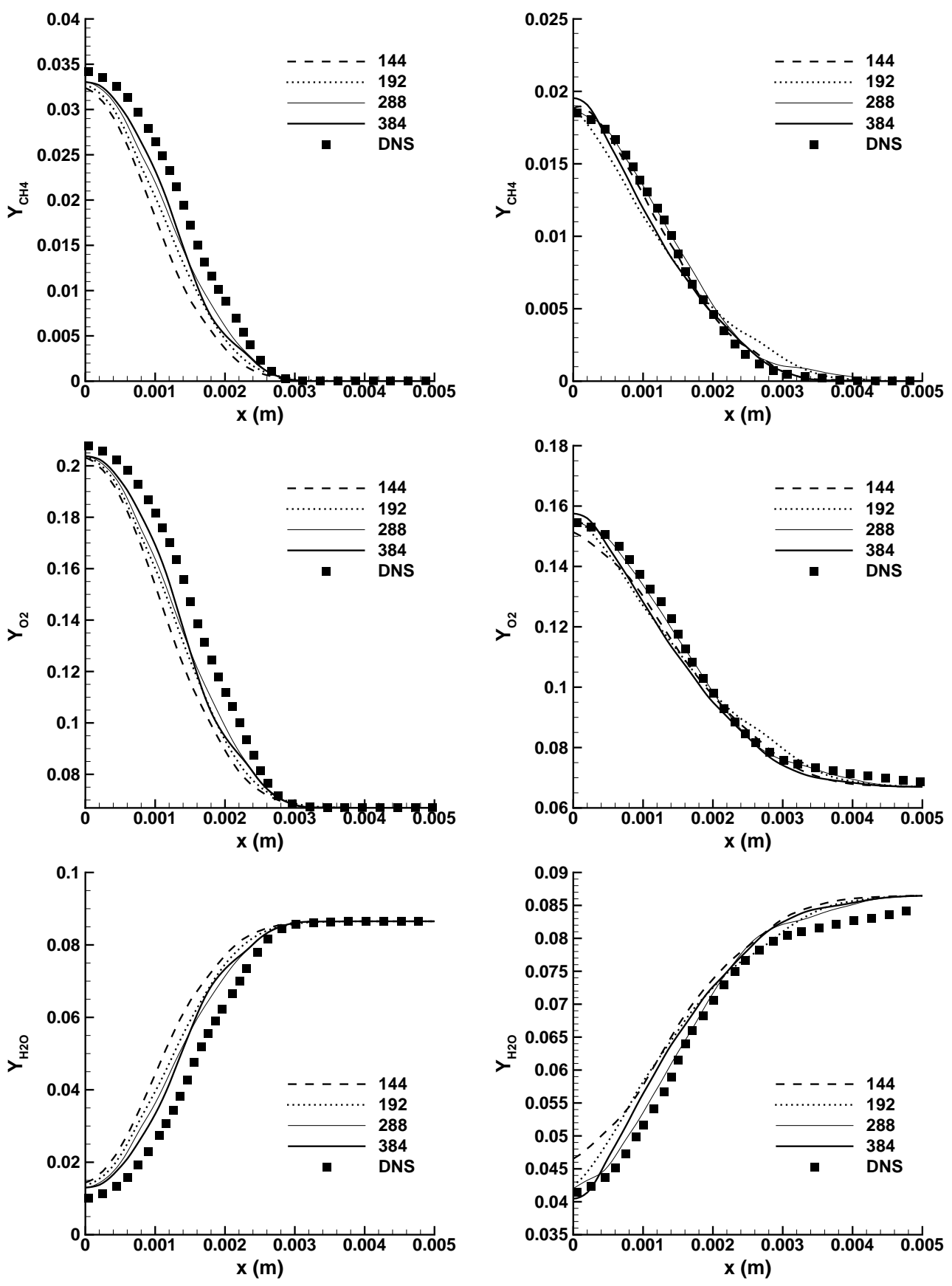

Figure 7: 

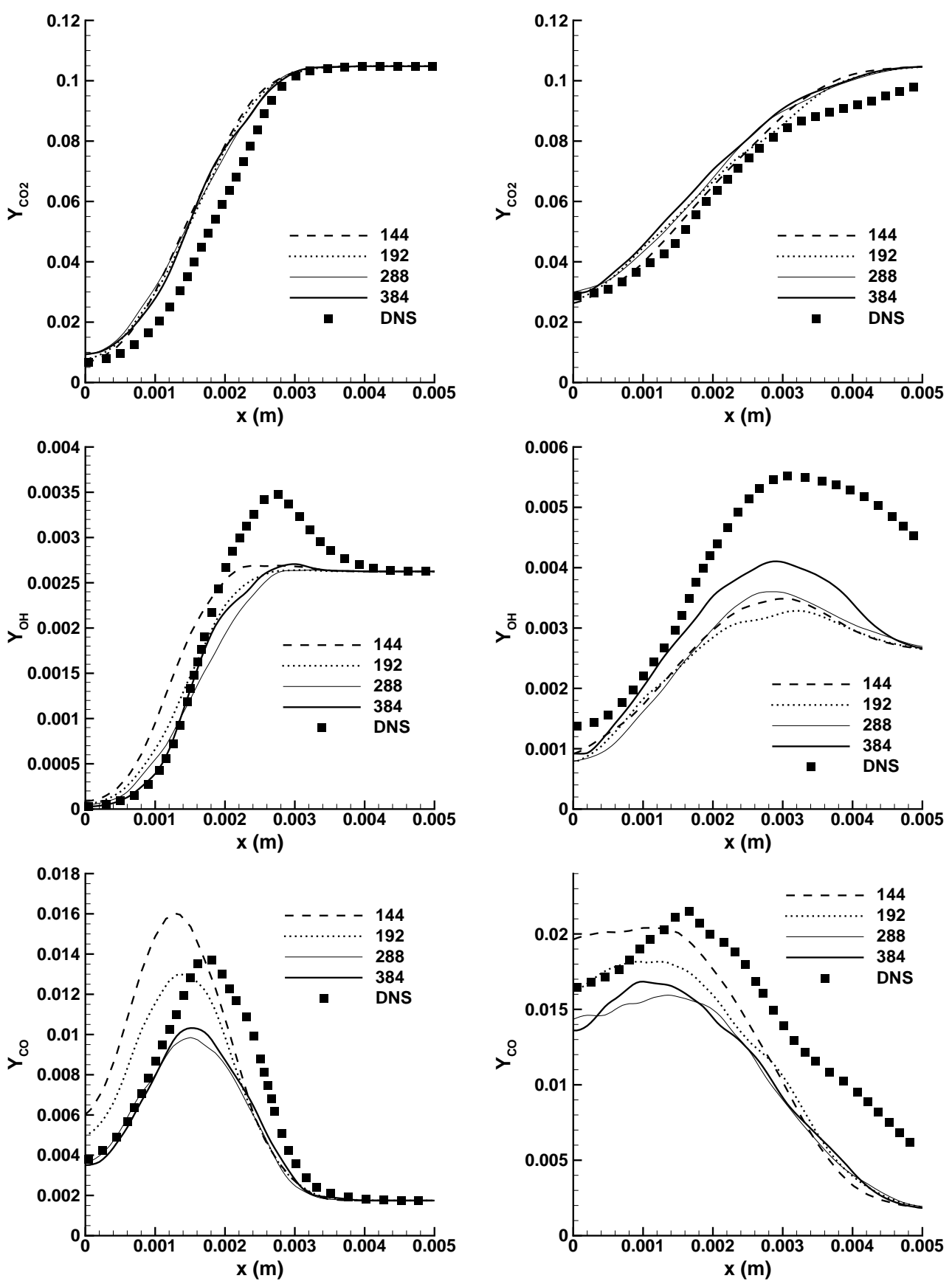

Figure 8: 

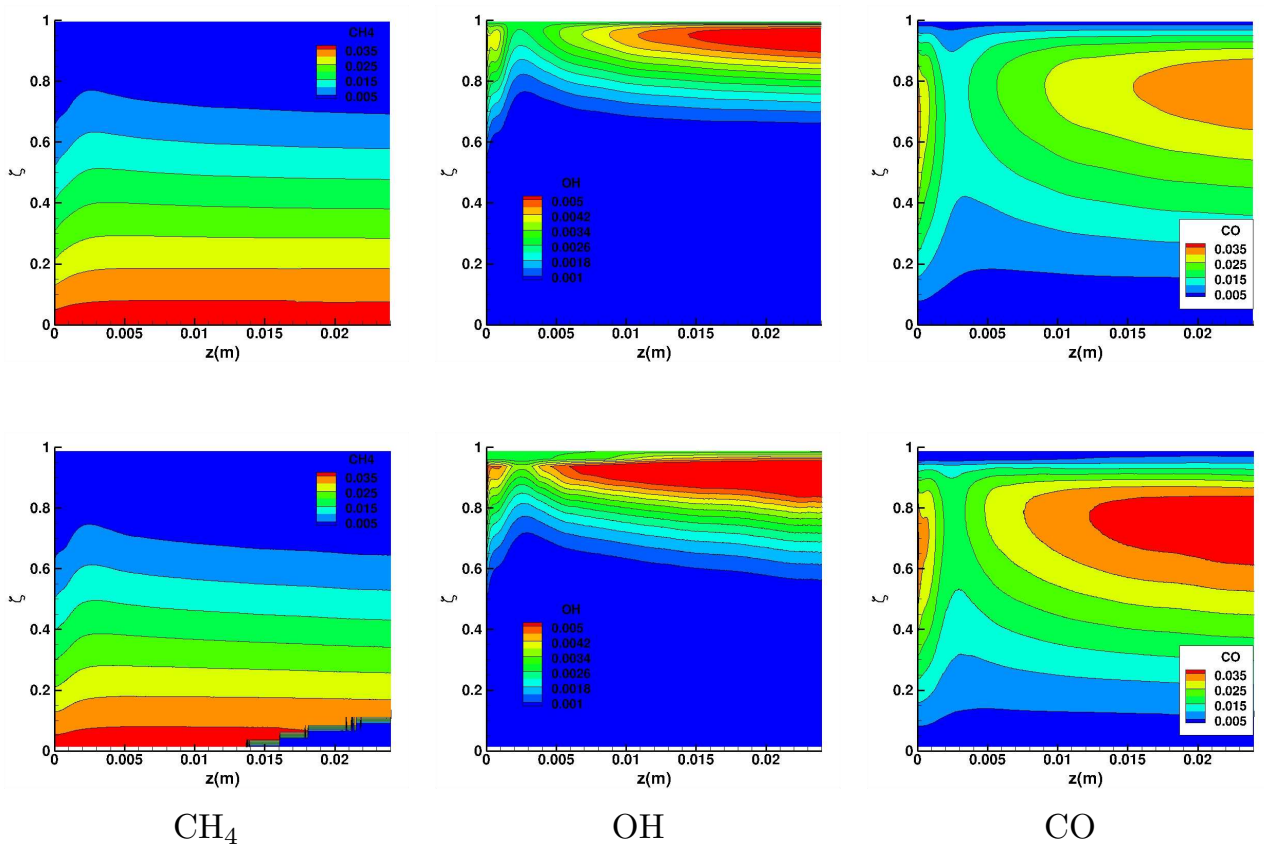

Figure 9: 


\section{Captions}

Figure 1: Instantaneous visualisation of absolute progress variable gradient(left) and isosurface of $\tilde{c}=0.65$ (right)

Figure 2: Comparisons of contours of the LES time averaged progress variable (left half of each plot) with DNS (right half of each plot). Contour lines run from $\tilde{c}=0.1$ to $\tilde{c}=0.9$ at intervals of $\Delta \tilde{c}=0.1$.

Figure 3: Comparisons of contours of the LES time averaged progress variable (left half of each plot) with DNS (right half of each plot) for the multiple physical dimension CMC model. Contour lines run from $\tilde{c}=0.1$ to $\tilde{c}=0.9$ at intervals of $\Delta \tilde{c}=0.1$.

Figure 4: Instantaneous visualisation of absolute progress variable gradient at $0.5 \mathrm{~ms}$ for the one dimensional, two dimensional and three dimensional CMC grids

Figure 5: Cross-stream variation of density and temperature taken at $z=$ $0.005 \mathrm{~m}$ (left) and $z=0.015 \mathrm{~m}$ (right)

Figure 6: Cross-stream variation of velocities $(\tilde{u}-x$ and $\tilde{w}-z$ direction) taken at $z=0.005 \mathrm{~m}$ (left) and $z=0.015 \mathrm{~m}$ (right)

Figure 7: Cross-stream variation of unconditionally averaged mass fractions taken at $z=0.005 \mathrm{~m}$ (left) and $z=0.015 \mathrm{~m}$ (right)

Figure 8: Cross-stream variation of unconditionally averaged mass fractions taken at $z=0.005 \mathrm{~m}$ (left) and $z=0.015 \mathrm{~m}$ (right)

Figure 9: Contour plots showing averaged conditionally filtered mass fractions from LES (top) and DNS (bottom) as a function of axial position. Note that a zero value is given in the DNS results where the conditional bins were empty. 


\section{Appendix A. Derivation of the CMC Equations for Premixed Com- bustion}

The derivation of the CMC equations for LES of premixed combustion follows the steps outlined by Navarro \& Kronenburg [13] with the difference that the equation for the progress variable now contains a source term. The conditional filtering procedure is defined using a fine grained Probability Density Function (PDF) $\psi \mid \zeta=\psi_{\zeta}=\delta[\zeta-c(\mathbf{x}, t)]$ where $\delta$ is the Dirac delta function, and $\zeta$ is the sample space of the progress variable $c$ defined as

$$
c=\frac{Y_{O_{2}, u}-Y_{O_{2}}(\mathbf{x}, t)}{Y_{O_{2}, u}-Y_{O_{2}, b}}
$$

where $Y_{\mathrm{O}_{2}}$ indicates the mass fraction of $\mathrm{O}_{2}$ and the subscripts $b$ and $u$ are the fully burnt and unburnt mass fractions. The evolution of $c$ is governed by the following transport equation:

$$
\frac{\partial \rho c}{\partial t}+\nabla \cdot(\rho u c)=\nabla \cdot\left(\rho D_{c} \nabla\right) c+\rho \dot{S}_{c}
$$

where $S_{c}=\dot{w}_{O_{2}} /\left(Y_{O_{2}, u}-Y_{O_{2}, b}\right)$. The conditional filtered value of the scalar $\Phi$ is then defined as [70]:

$$
\overline{\Phi \mid \zeta}=\frac{\int_{V} \Phi \psi_{\zeta}\left(c\left(\mathbf{x}^{\prime}, t\right)-\zeta\right) G\left(\mathbf{x}-\mathbf{x}^{\prime}, \Delta\right) d V^{\prime}}{\bar{P}(\zeta)}
$$

where $G$ is a space filter of width $\Delta, V$ is the volume of the computational domain, and $\bar{P}(\zeta)$ is the Filtered Probability Density Function (FDF) defined as $\bar{P}(\zeta)=\int_{V} \psi_{\zeta}\left(c\left(\mathbf{x}^{\prime}, t\right)-\zeta\right) G\left(\mathbf{x}-\mathbf{x}^{\prime}, \Delta\right) d V^{\prime}$. Favre filtering is employed here where $\overline{\rho|\zeta \Phi| \zeta}=\overline{\rho \Phi \mid \zeta}$, and the Favre filtered FDF is denoted $\tilde{P}(\zeta)$. The transport equations for the fine grained PDF and the progress variable can be written as [11]:

$$
\begin{array}{r}
\frac{\partial \rho \psi_{\zeta}}{\partial t}+\nabla \cdot \rho \mathbf{u} \psi_{\zeta}=-\frac{\partial^{2}}{\partial \zeta^{2}}\left(\rho N \psi_{\zeta}\right)-\frac{\partial}{\partial \zeta} \nabla \cdot\left(\rho \psi_{\zeta} D_{c} \nabla c\right)-S_{c} \frac{\partial \rho \psi_{\zeta}}{\partial \zeta} \\
\frac{\partial \rho \Phi \psi_{\zeta}}{\partial t}+\nabla \cdot \rho \mathbf{u} \Phi \psi_{\zeta}=-\frac{\partial^{2}}{\partial \zeta^{2}}\left(\rho N \Phi \psi_{\zeta}\right)-\frac{\partial}{\partial \zeta} \Phi \nabla \cdot\left(\rho \psi_{\zeta} D_{c} \nabla c\right)+ \\
\psi \nabla \cdot\left(\rho \Phi \psi_{\zeta} D_{\Phi} \nabla \Phi\right)+\psi_{\zeta} S_{\Phi}-S_{c} \frac{\partial \rho \Phi \psi_{\zeta}}{\partial \zeta}
\end{array}
$$


where the scalar dissipation $N=D_{\Phi}(\nabla \Phi)^{2}$, and $S_{\Phi}$ is the scalar source term. These equations are equivalent to Equations (40) and (41) in [13]. The next step is to multiply Equations (A.4) and (A.5) by the filter function $G$ which commutes with spatial and temporal differentiation (constant spacing assumed), and integrating over the physical domain $V^{\prime}$ to give the FDF transport equation and the conditional scalar transport equation. For this derivation it is assumed that the Lewis number is unity for all species (i.e. no differential diffusion effects). The only difference to the derivation for nonpremixed LES-CMC is the final term on the right hand side of Equations (A.4) and (A.5), hence this term is detailed here. When multiplied by $G$ and integrated these terms are transformed as follows:

$$
\begin{aligned}
\int_{V} S_{c} \frac{\partial \rho \psi_{\zeta}}{\partial \zeta} G d V^{\prime}= & \int_{V} \frac{\partial}{\partial \zeta}\left(\rho \psi_{\zeta} S_{c} G\right) d V^{\prime}-\int_{V} \rho \psi_{\zeta} \frac{\partial S_{c}}{\partial \zeta} G d V^{\prime} \\
& \rightarrow \frac{\partial}{\partial \zeta}\left(\overline{\rho \mid \zeta} \widetilde{S_{c} \mid \zeta} \tilde{P}(\zeta)\right)-\overline{\rho \mid \zeta} \tilde{P}(\zeta) \widehat{\frac{\partial S_{c}}{\partial \zeta} \mid \zeta} \\
\int_{V} S_{c} \frac{\partial \rho \Phi \psi_{\zeta}}{\partial \zeta} G d V^{\prime} & =\int_{V} \frac{\partial}{\partial \zeta}\left(\rho \Phi \psi_{\zeta} S_{c} G\right) d V^{\prime}-\int_{V} \rho \Phi \psi_{\zeta} \frac{\partial S_{c}}{\partial \zeta} G d V^{\prime} \\
\rightarrow & \frac{\partial}{\partial \zeta}\left(\overline{\rho \mid \zeta}\left(\widetilde{\left.S_{c} \Phi\right) \mid \zeta} \tilde{P}(\zeta)\right)-\overline{\rho \mid \zeta} \tilde{P}(\zeta)\left(\frac{\partial S_{c}}{\partial \zeta}\right) \mid \zeta .\right.
\end{aligned}
$$

The derivation employed by Navarro \& Kronenburg [13] holds for the filtering of the remaining terms in Equations (A.4) and (A.5), where the RANS-CMC linear diffusion approximation is invoked. The FDF transport equation is

$$
\begin{aligned}
& \frac{\partial \overline{\rho \mid \zeta} \tilde{P}(\zeta)}{\partial t}+\nabla \cdot(\overline{\rho \mid \zeta} \widetilde{\mathbf{u} \mid \zeta} \tilde{P}(\zeta))=-\frac{\partial^{2} \overline{\rho \mid \zeta} \widetilde{N \mid \zeta} \tilde{P}(\zeta)}{\partial \zeta^{2}} \\
& -\frac{\partial}{\partial \zeta}\left[\nabla \cdot \overline{\rho \mid \zeta}(\widetilde{D \nabla c) \mid} \zeta \tilde{P}(\zeta)]+\frac{\partial}{\partial \zeta}\left(\overline{\rho \mid \zeta} \widetilde{S_{c} \mid \zeta} \tilde{P}(\zeta)\right)-\overline{\rho \mid \zeta} \tilde{P}(\zeta) \widetilde{\frac{\partial S_{c}}{\partial \zeta} \mid \zeta,}\right.
\end{aligned}
$$

and transformation of the scalar transport equation yields, 


$$
\begin{aligned}
\left.\frac{\partial \widetilde{\rho|\zeta \Phi| \zeta} \tilde{P}(\zeta)}{\partial t}+\nabla \cdot \overline{(\rho \mid \zeta} \widetilde{(\mathbf{u} \Phi) \mid \zeta} \tilde{P}(\zeta)\right)=\widetilde{S_{\Phi} \mid \zeta}+\nabla \cdot f_{D}+ \\
\overline{\rho \mid \zeta \tilde{N \mid \zeta}} \tilde{P}(\zeta) \frac{\partial^{2} \Phi \mid \zeta}{\partial \zeta^{2}}-\frac{\partial^{2} \overline{\rho \mid \zeta} \widetilde{N \mid \zeta} \tilde{P}(\zeta)}{\partial \zeta^{2}} \widetilde{\Phi \mid \zeta}+ \\
\\
\frac{\partial}{\partial \zeta}\left(\overline{\rho \mid \zeta} \widetilde{\left(S_{c} \Phi\right) \mid \zeta} \tilde{P}(\zeta)\right)-\overline{\rho \mid \zeta} \tilde{P}(\zeta)\left(\widetilde{\left.\frac{\partial S_{c}}{\partial \zeta} \Phi\right) \mid}, \zeta\right.
\end{aligned}
$$

where,

$$
f_{D}=\overline{\rho \mid \zeta} \tilde{P}(\zeta)\left(\widetilde{D \nabla \Phi) \mid \zeta}-\frac{\partial}{\partial \zeta}[\overline{\rho \mid \zeta} \tilde{P}(\zeta)(\widetilde{D \nabla c \Phi}) \mid \zeta] .\right.
$$

To simplify the form of the resultant filtered transport equations Eqn. (A.8) is multiplied by $\widetilde{\Phi \mid \zeta}$ and then subtracted from Eqn. (A.9). The terms arising due to the choice of a reactive scalar as a progress variable then simplify, as Eqn. (A.7) minus Eqn. (A.6) $\times \widetilde{\Phi \mid \zeta}$ gives:

$$
\begin{aligned}
& \frac{\partial}{\partial \zeta}\left(\overline{\rho \mid \zeta} \widetilde{\left.S_{c} \Phi\right) \mid \zeta} \tilde{P}(\zeta)\right)-\overline{\rho \mid \zeta} \tilde{P}(\zeta)\left(\widetilde{\left.\frac{\partial S_{c}}{\partial \zeta} \Phi\right) \mid}-\right. \\
& {\left[\widetilde{\Phi \mid \zeta} \frac{\partial}{\partial \zeta}\left(\overline{\rho \mid \zeta} \widetilde{S_{c} \mid \zeta} \tilde{P}(\zeta)\right)-\widetilde{\Phi \mid \zeta \overline{\rho \mid \zeta}} \tilde{P}(\zeta) \frac{\partial S_{c}}{\partial \zeta} \mid \zeta\right] \rightarrow \overline{\rho \mid \zeta} \widetilde{S_{c} \mid \zeta} \tilde{P}(\zeta) \frac{\partial \widetilde{\Phi \mid \zeta}}{\partial \zeta}}
\end{aligned}
$$

The resultant form of the conditional scalar transport equation can be written as:

$$
\frac{\partial \widetilde{\Phi \mid \zeta}}{\partial t}+\widetilde{\mathbf{u} \mid \zeta} \cdot \nabla \widetilde{\Phi \mid \zeta}=\frac{\widetilde{S_{\Phi} \mid \zeta}}{\overline{\rho \mid \zeta}}-\widetilde{S_{c} \mid \zeta} \frac{\partial \widetilde{\Phi \mid \zeta}}{\partial \zeta}+\widetilde{N \mid \zeta} \frac{\partial^{2} \widetilde{\Phi \mid \zeta}}{\partial \zeta}+e_{\Phi}+e_{D}
$$

where $e_{\Phi}$ and $e_{D}$ are defined as:

$$
\overline{\rho \mid \zeta} \tilde{P}(\zeta) e_{\Phi}=\nabla \cdot[\overline{\rho \mid \zeta}(\widetilde{\mathbf{u}|\zeta \Phi| \zeta}-\widetilde{(\mathbf{u} \Phi) \mid \zeta}) \tilde{P}(\zeta)],
$$

and 


$$
\begin{aligned}
\overline{\rho \mid \zeta} \tilde{P}(\zeta) e_{D}=\nabla \cdot \overline{\rho \mid \zeta} \tilde{P}(\zeta)(\widetilde{D \nabla \Phi) \mid \zeta}- & \frac{\partial}{\partial \zeta} \cdot \nabla[\overline{\rho \mid \zeta} \tilde{P}(\zeta)(\widetilde{D \nabla c) \mid \zeta}]+ \\
& \widetilde{\Phi \mid \zeta} \frac{\partial}{\partial \zeta}[\nabla \cdot \overline{\rho \mid \zeta} \tilde{P}(\zeta)(\widetilde{D \nabla c) \mid}]
\end{aligned} .
$$

In this study $e_{D}$ is neglected as it is expected to tend to zero as $R e \rightarrow \infty$. Here the scalars solved for are the conditional mass fractions $Q=\widetilde{Y_{k} \mid \zeta}$, and defining $\widetilde{\dot{w}_{k} \mid \zeta}=\left(\widetilde{S_{Y_{k}} \mid \zeta}\right) /(\overline{\rho \mid \zeta})$ the final form of the premixed LES-CMC equations is:

$$
\frac{\partial Q}{\partial t}+\widetilde{\mathbf{u} \mid \zeta} \cdot \nabla Q=\widetilde{\dot{w}_{k} \mid \zeta}-\widetilde{S_{c} \mid \zeta} \frac{\partial Q}{\partial \zeta}+\widetilde{N \mid \zeta} \frac{\partial^{2} Q}{\partial \zeta}+e_{Q}
$$

